\title{
DIFFUSE GALACTIC GAMMA-RAY EMISSION
}

\section{Hans Bloemen}

Leiden Observatory, P.O. Box 9513, 2300 RA Leiden, The Netherlands

\section{INTRODUCTION}

This review concentrates on gamma radiation that originates from cosmicray (CR) interactions with gas and photons in the interstellar medium (ISM). The $\gamma$-ray energy band of interest here ranges from $\sim 1 \mathrm{MeV}$ to $\sim 100 \mathrm{GeV}$, but this work is restricted to the energy interval between about $50 \mathrm{MeV}$ and $5 \mathrm{GeV}$ (generally referred to as "high-energy" $\gamma$ rays), which is mainly dictated by data availability. The experimental development is most advanced at these energies. Also, high-energy $\gamma$ rays are of particular interest from an astrophysical point of view because they are partly produced by the CR particles that carry the bulk of the CR energy. The feasibility of detecting high-energy $\gamma$ quanta from the ISM was predicted over 35 years ago $(101,120)$, but the experimental development of $\gamma$ ray astronomy has been slow because space-borne telescopes with high technical requirements are needed in order to detect the rare $\gamma$ rays in a high background of CR particles. After several pioneering balloon and a few satellite experiments [reviewed by Fazio (80)], a real breakthrough in observational $\gamma$-ray astronomy was achieved with the NASA satellite $S A S-2$ (83), followed soon after by the ESA satellite $C O S$ - $B$. Since mid 1982, when the $C O S-B$ experiment was switched off, no high-energy $\gamma$-ray satellite has been operative, and it may not be obvious at first glance that considerable progress has been made in this field recently. This article emphasizes new results obtained from analyses of the final $C O S-B$ database (168). Descriptions of the instrument and of the prelaunch and inflight calibrations can be found in $(21,31,109,209,233)$.

Both $S A S-2$ and $C O S-B$ viewed essentially the entire Galactic disk up to $|b| \approx 20^{\circ}$, with several extensions to higher latitudes. The characteristics of the two instruments were not vastly different (geometrical area of the detector $\sim 500 \mathrm{~cm}^{2}$, field of view $\sim 0.3 \mathrm{sr}$, average angular resolution 
above $100 \mathrm{MeV} \sim 2.5^{\circ} \mathrm{FWHM}$ ), but the counting statistics of the $C O S-B$ observations are higher by a factor of $\sim 25$ owing to the long duration of the $C O S-B$ mission - namely, about $6 \frac{1}{2}$ years compared with about 7 months for $S A S-2$. In addition, $C O S-B$ could measure energies beyond the approximate $200-\mathrm{MeV}$ upper limit of the $S A S-2$ instrument. These advantages have been fully exploited in recent analyses. On the other hand, the $S A S$-2 observations have a lower instrumental background owing to a differing orbit of the satellite, which is important for studies of the low-intensity emission observed at high latitudes. Figure 1 shows a $C O S-B$ map of the Milky Way for the energy range $100 \mathrm{MeV}-6 \mathrm{GeV}$, which is best suited for pictorial purposes because it gives a good compromise between counting statistics and angular resolution (which degrades with decreasing energy).

During the 10-yr time interval between the first $C O S-B$ observations and the release of the final data base, our understanding of the instrument and the data has clearly improved in several aspects. Not surprisingly, the results have evolved. I have included here some unpublished updates and extensions of earlier work. All figures were extracted from the final $\mathrm{COS}$ $B$ data base.

Specific aspects or more general properties of diffuse gamma radiation have been reviewed in the monographs by Stecker in 1971 (222), Chupp in 1976 (61), Fichtel \& Trombka in 1981 (90), Ginzburg in 1984 (93), and Ramana Murthy \& Wolfendale in 1986 (196) and in the review papers by Fazio in 1967 (79), Fichtel in 1977 (82), Van der Walt \& Wolfendale in 1988 (246) and Dogiel \& Ginzburg in 1989 (72). In the 1983 volume of this series, Bignami \& Hermsen (24) have reviewed the $\gamma$-ray point sources that have been detected.

In Section 2, some basic principles are described and the main observational results are summarized. The interpretation of these findings is discussed further in Sections 3 and 4. Two important types of inference can be distinguished: the relevance to molecular-gas studies (Section 3), and the relevance to CR studies (Section 4). In Section 5 the status regarding $\gamma$-ray point sources is briefly discussed, with emphasis on their possibly diffuse nature.

Unless stated otherwise, the radius of the solar circle is assumed to be $10 \mathrm{kpc}$.

\section{GAMMA RAYS AND THE INTERSTELLAR MEDIUM}

\subsection{Gamma-Ray Emission Processes}

Radiative transfer in $\gamma$-ray astronomy is relatively simple because the Galaxy is practically transparent to $\gamma$ rays with energies up to $\sim 10^{14} \mathrm{eV}$. 

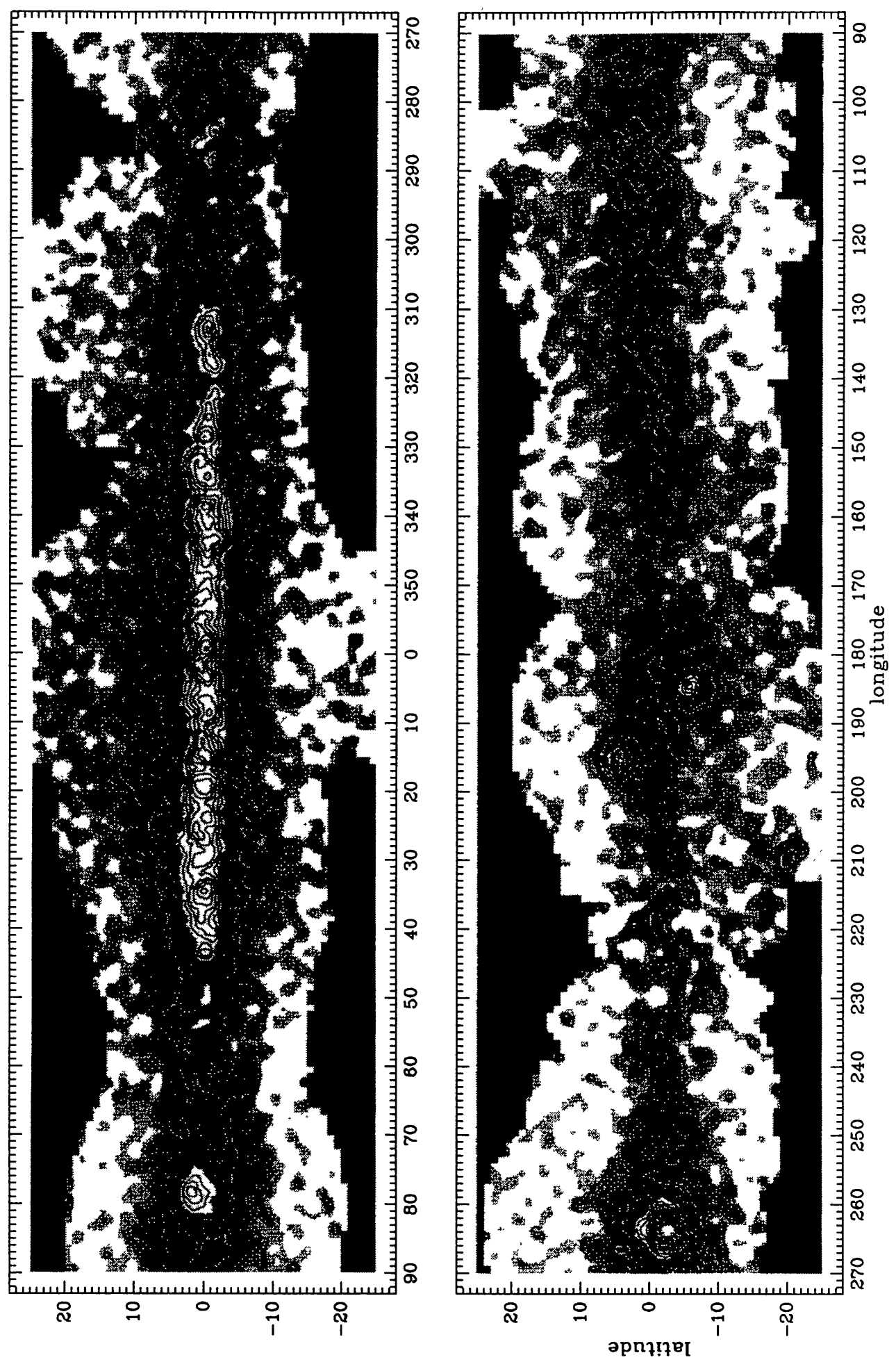

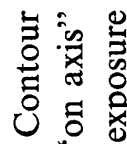

足

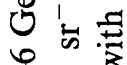

> in

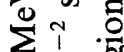

읭

용

응

जू

정응

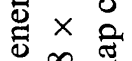

$\underset{\square}{\infty}$

봉

远

-

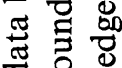

$\infty$ bo

它

0 .

西

돈

용

至是焉

它远

। 焉.

各的葛

पै के

है

용

해용

0 造 n

䎹

○ $\times$ 을

色的 营

岁范

离过焉

¿.

. .

芯芯边

胥 ఫ્屯

牙

ชั่ 듕 응

- 0

¿ సै

s

逭过 
The $\gamma$-ray production mechanisms related to CR interactions with the ISM are well understood and have been described in detail by a number of authors [e.g. in the monographs by Chupp (61) and Stecker (222)]. The important production processes for the $50 \mathrm{MeV}-5 \mathrm{GeV}$ range are $\pi^{\circ}$ decay, bremsstrahlung, and inverse-Compton (IC) scattering, of which a brief description is given below. The $\gamma$-ray emissivity per unit of volume is denoted by $Q_{\gamma}$, and the emissivity per $\mathrm{H}$ atom by $\varepsilon_{\gamma} \equiv Q_{\gamma} / 4 \pi n$ [photon $(\mathrm{H}$ atom) $)^{-1} \mathrm{~s}^{-1} \mathrm{sr}^{-1}$, where $n$ is the number density of $\mathrm{H}$ atoms (or, more precisely in case the gas is ionized, hydrogen nuclei). The quantity $\varepsilon_{\gamma}$ is, of course, particularly meaningful when the $\gamma$-ray emission is directly related to the gas particles, which is the case for the $\pi^{\circ}$-decay and bremsstrahlung processes.

(a) Nuclear interactions ( $\pi^{\circ}$-decay) Nuclear interactions between CR particles and nuclei of the interstellar gas lead via various decay chains $(69$, $223,227)$ to the production of $\pi^{\circ}$-mesons, which decay rapidly $\left(\sim 10^{-16} \mathrm{~s}\right)$ and with a probability of almost $100 \%$ into two $\gamma$ quanta. The bulk of the $\pi^{\circ}$-mesons is produced by those cosmic rays that carry most of the CR energy density, i.e. $\mathrm{CR}$ protons with kinetic energies between about $1 \mathrm{GeV}$ and a few tens of GeV. (The term "protons" instead of "nuclei" is therefore mostly used throughout this paper.) Each $\gamma$ quantum has an energy of $m_{\pi^{\circ}} c^{2} / 2 \approx 68 \mathrm{MeV}$ in the rest frame of the $\pi^{\circ}$-meson (where $m_{\pi^{\circ}}$ is the mass of the $\pi^{\circ}$ meson), which transforms into a broad energy distribution centered on $\sim 68 \mathrm{MeV}$ in the observer's reference system (see Figure 2). At $\gamma$-ray energies above $\sim 1 \mathrm{GeV}$, the shape of the $\pi^{\circ}$-decay $\gamma$-ray spectrum converges to that of the $\mathrm{CR}$ proton spectrum at energies above a few $\mathrm{GeV}$. More specifically, if the differential energy spectrum of the CR protons is a power-law spectrum, $I_{\mathrm{p}}\left(E_{\mathrm{p}}\right)=K_{\mathrm{p}} E_{\mathrm{p}}^{-\Gamma}$ (where $K_{\mathrm{p}}$ is a normalization factor), then the differential emissivity spectrum of the $\pi^{\circ}$-decay $\gamma$ rays converges to a similar power-law spectrum, $Q_{\gamma}\left(E_{\gamma}\right) \propto n K_{\mathrm{p}} E_{\gamma}^{-\Gamma}$, where $n$ is the density of the target gas nuclei.

(b) Bremsstrahlung Coulomb scattering of CR electrons on the nuclei and electrons of the interstellar gas leads to the production of bremsstrahlung $\gamma$ rays. At a particular $\gamma$-ray energy the bremsstrahlung emission originates predominantly from those CR electrons that have energies in the range of about one decade immediately above the $\gamma$-ray energy, so that $E_{\mathrm{e}} \approx 3 E_{\gamma}$. The $\gamma$-ray emissivity spectrum can be written as $Q_{\gamma}\left(E_{\gamma}\right) \propto$ $n I_{\mathrm{e}}\left(>E_{\gamma}\right) / E_{\gamma}$, so for a power-law electron spectrum of the form $I_{\mathrm{e}}\left(E_{\mathrm{e}}\right)=$ $K_{\mathrm{e}} E_{\mathrm{e}}^{-\Gamma}$, the production spectrum is a similar power-law spectrum $Q_{\gamma}\left(E_{\gamma}\right) \propto n\left[K_{\mathrm{e}} /(\Gamma-1)\right] E_{\gamma}^{-\Gamma}$, where $n$ is the density of the gas nuclei. For the energetic electrons that are of interest here $\left(E_{\mathrm{e}} \gtrsim 100 \mathrm{MeV}\right)$, screening by electrons in the target atoms plays a role in the scattering process (41). 


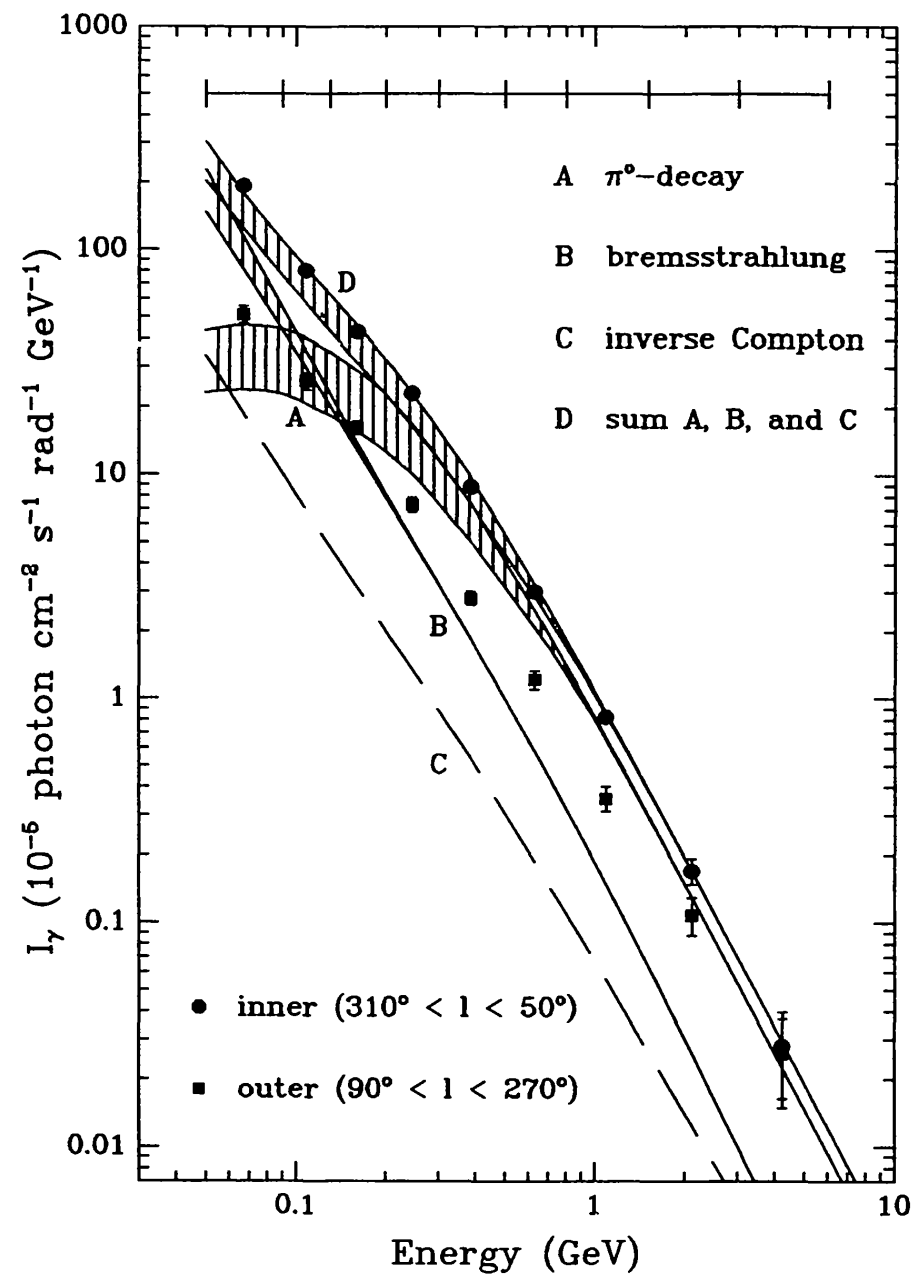

Figure 2 Spectra of the $\gamma$-ray emission in the general directions of the inner and the outer Galaxy $\left(|b|<30^{\circ}\right)$ as observed by the $\operatorname{COS}-B$ satellite, and spectra derived from the local interstellar CR electron and proton spectra (33). The sum of the spectra for the three individual $\gamma$-ray production processes is normalized to the observed inner-Galaxy spectrum at $\sim 1 \mathrm{GeV}$. The shaded areas indicate uncertainties in the predictions that result largely from uncertainties in the corrections for solar modulation of the observed CR spectra (Section 2.2). The hint for a flatter $\gamma$-ray spectrum in the outer-Galaxy direction is discussed at the end of Section 2.3.

Consequently, the bremsstrahlung emissivity for electrons incident on an atomic gas is not identical to that for electrons incident on an ionized gas. In addition, electron-electron scattering needs to be taken into account in the latter case; the bremsstrahlung cross sections for an electron incident on an unshielded free proton and an unshielded free electron are roughly equal (96). The relevant parameter here is the so-called screening factor, given by $\Delta=68.5 m_{\mathrm{e}} c^{2} E_{\gamma} /\left[2 E_{\mathrm{e}}\left(E_{\mathrm{e}}-E_{\gamma}\right)\right]$ for atomic hydrogen; screening is important if $\Delta \ll 1$, which is the case for high-energy electrons. At $E_{\gamma}=100$ $\mathrm{MeV}$, for example, $\Delta \approx 0.03$ and the total $\varepsilon_{\gamma}$ for ionized hydrogen (includ- 
ing electron-electron scattering) is about $30 \%$ higher than $\varepsilon_{\gamma}$ for atomic hydrogen (41). Although the effect is small and in fact negligible for studies of the Galactic disk, where the fraction of the gas that is in ionized form is on average small, it may be an important clue for studies of mediumlatitude regions (Section 2.4).

(c) Inverse Compton The scattering of high-energy $\left(E_{\mathrm{e}} \gtrsim 10 \mathrm{GeV}\right) \mathrm{CR}$ electrons on soft photons of energy $\varepsilon \ll m_{\mathrm{e}} c^{2}$ (mainly optical and infrared photons and the $2.7-\mathrm{K}$ universal background radiation) leads to the production of IC $\gamma$ rays. If the incident electrons have a power-law spectrum, $I_{\mathrm{e}}\left(E_{\mathrm{e}}\right)=K_{\mathrm{e}} E_{\mathrm{e}}^{-\Gamma}$, then the IC emissivity has approximately a power-law spectrum of the form $Q_{\gamma}\left(E_{\gamma}\right) \propto n_{\mathrm{ph}}\langle\varepsilon\rangle^{(\Gamma-1) / 2} K_{\mathrm{e}} E_{\gamma}^{-(\Gamma+1) / 2}$, where $\langle\varepsilon\rangle$ and $n_{\mathrm{ph}}$ are the average energy of the target photons and their number density, respectively. For the $\gamma$-ray energies of interest here, the mean energy of the produced $\gamma$ quantum is given by $\left\langle E_{\gamma}\right\rangle \approx \frac{4}{3} \gamma^{2}\langle\varepsilon\rangle$, where $\gamma=E_{\mathrm{e}} / m_{\mathrm{e}} c^{2}$. For example, at $\left\langle E_{\gamma}\right\rangle=100 \mathrm{MeV}$, electrons with very high energies of typically $E_{\mathrm{e}} \approx 200 \mathrm{GeV}$ are responsible for IC emission from the $2.7-\mathrm{K}$ background $\left(\langle\varepsilon\rangle \approx 6 \times 10^{-4} \mathrm{eV}\right)$.

A few general remarks can be made based on the above.

First, the volume emissivities $Q_{\gamma}$ of the three $\gamma$-ray production processes are proportional to the CR density (in a certain energy range) and to either the gas density or the photon density. The $\gamma$-ray intensity $I_{\gamma}$ can be written as $\int Q_{\gamma}\left(E_{\gamma}, \mathbf{r}\right) / 4 \pi d \mathbf{r}$, where the integration is along the line of sight, or

$$
I_{\gamma}\left(E_{\gamma}\right)=\frac{1}{4 \pi} \int_{l} d \mathbf{r} n(\mathbf{r}) \int_{E_{\gamma}}^{\infty} d E \sigma\left(E_{\gamma}, E\right) I_{\mathrm{CR}}(E, \mathbf{r}),
$$

where $\sigma\left(E_{\gamma}, E\right)$ is the cross section of the process, and $n(\mathbf{r})$ is the gas or photon density at position $\mathbf{r}$. Following this notation, the $\gamma$-ray emissivity per $\mathrm{H}$ atom for the $\pi^{\circ}$-decay and bremsstrahlung processes is given by

$$
\varepsilon_{\gamma}\left(E_{\gamma}, \mathbf{r}\right)=\frac{1}{4 \pi} \int_{E_{\gamma}}^{\infty} d E \sigma\left(E_{\gamma}, E\right) I_{\mathrm{CR}}(E, \mathbf{r}) .
$$

Second, the relative importance of the emission processes is a strong function of $\gamma$-ray energy. The most distinct change of importance occurs around $100 \mathrm{MeV}$, which is evident from Figure 2; the relative contributions of the bremsstrahlung and IC processes increase strongly with decreasing energy because they have roughly power-law emissivity spectra, whereas the $\pi^{\circ}$-decay spectrum shows the characteristic bump. In addition, for $E_{\gamma} \gtrsim 1 \mathrm{GeV}$, the importance of IC emission can be expected to increase. The reason for this is that the IC emissivity follows approximately an $E_{\gamma}^{-2}$ power law [at least in the solar vicinity, to which our knowledge of 
the high-energy electron spectrum is limited: $\Gamma \approx 3-3.4$ for $E_{\mathrm{e}} \gtrsim 100 \mathrm{GeV}$ $(179,180)]$, whereas the $\pi^{\circ}$-decay and bremsstrahlung emissivities are both approximately proportional to $E_{\gamma}^{-2.7}[\Gamma \approx 2.7$ for the local interstellar electron spectrum at $\mathrm{GeV}$ energies (e.g. 250) and for the proton spectrum above $10 \mathrm{GeV}$ (e.g. 206)].

Third, the relative contributions of the three processes can be expected to be a function of position in the Galaxy. Most obvious is the impact of different distributions of interstellar matter and soft photons, which lead to spatial variations of the contribution from CR-photon and CR-matter interactions. Another reason for variations of the contributions is the production of so-called secondary electrons by the interaction of the CR proton-nuclear component with interstellar gas, which may lead to enhanced bremsstrahlung emission in high-density regions (such as molecular clouds). Finally, a variety of effects influences the spectral distribution of CR particles during propagation and hence, possibly, the relative contributions of the $\gamma$-ray production processes.

\subsection{Gamma-Ray Intensity Versus Gas Column Density}

Several authors have reached the conclusion that the IC contribution to the measured $\gamma$-ray intensities near the Galactic plane does not exceed 5$10 \%(25,32,131,191,214,224)$. As an example, Figure 2 (from 33) shows estimates of the relative importance of the three main emission processes in the general direction of the inner Galaxy. For further discussions in this paper it is illustrative to know how these estimates were obtained. Only the IC spectrum in the figure is a "real" prediction of absolute intensities (32). It is based on a variety of studies of the interstellar radiation field; the density of high-energy electrons was assumed to increase toward the inner Galaxy (a factor of two between $R=10$ and $5 \mathrm{kpc}$ - see Section 4.3) and to have a spectral shape as measured near Earth and a scale height of $750 \mathrm{pc}$. Also, the shapes of the predicted $\pi^{\circ}$-decay and bremsstrahlung $\gamma$-ray spectra are based on measurements of the CR electron and proton spectra near Earth. The relative $\gamma$-ray intensities of these two production mechanisms are fixed (defined by the local CR electron/proton ratio), but the sum was normalized such that the total predicted $\gamma$-ray intensity at $1 \mathrm{GeV}$ is equal to that observed in the inner-Galaxy direction. The shaded areas indicate uncertainties in the predictions that result largely from uncertainties in the corrections for solar modulation of the observed CR spectra. The upper bound of the shaded area for the $\pi^{\circ}$-decay curve represents the spectrum calculated by Stephens \& Badhwar (227; their " $M_{\mathrm{U}}$ " curve), and the lower bound corresponds to the spectrum given by Dermer (69). The upper and lower bounds of the shaded area for the bremsstrahlung spectrum correspond to an $E_{\mathrm{e}}^{-2.4}$ and $E_{\mathrm{e}}^{-2.1}$ electron spec- 
trum for $E_{\mathrm{e}} \lesssim 200 \mathrm{MeV}$, respectively, which covers the uncertainties at these energies (250).

The IC contribution is expected to increase with increasing Galactic latitude because the scale height of the interstellar gas is smaller than that of optical photons and, of course, the isotropic $2.7-\mathrm{K}$ background. At $E_{\gamma} \gtrsim 1 \mathrm{GeV}$, the IC contribution may even be very substantial. Owing to uncertainties in the electron scale height, however, it is very hard to make a precise estimate. Further discussion on this point is given in Section 2.4.

The $\gamma$-ray intensity is a measure of the product of CR density and total gas density, integrated along the line of sight, if the emission can be largely attributed to CR-matter interactions. $I_{\gamma}$, for instance, is a measure of the total gas column density $N$, practically irrespective of the composition or the physical state of the gas, if the CR density can be assumed to be constant: $I_{\gamma}=\varepsilon_{\gamma} N$. Vice versa, the CR distribution can be studied if independent information on the gas distribution is available. Several $\gamma$-ray studies have used H I 21-cm line observations and millimeter-wave line observations of the $\mathrm{CO}$ molecule to trace the column densities of atomic $[N(\mathrm{H} \mathrm{I})]$ and molecular hydrogen $\left[N\left(\mathrm{H}_{2}\right)\right]$, respectively, and maps of selected sky areas were compared with the observed $\gamma$-ray intensity distributions. The relationship that is evaluated in these comparisons is of the form $I_{\gamma}=\varepsilon_{\gamma}\left\{N(\mathrm{H} \mathrm{I})+2 X W_{\mathrm{CO}}\right\}$, where $W_{\mathrm{CO}}$ is the velocity-integrated $\mathrm{CO}$ antenna temperature and $X \equiv N\left(\mathrm{H}_{2}\right) / W_{\mathrm{CO}}$ is the CO-to- $\mathrm{H}_{2}$ calibration factor in units of molecule $\mathrm{cm}^{-2}\left(\mathrm{~K} \mathrm{~km} \mathrm{~s}^{-1}\right)^{-1}$. Both $\varepsilon_{\gamma}$ and $X$ can be estimated from a correlation analysis of the $\mathrm{H} \mathrm{I}, \mathrm{CO}$, and $\gamma$-ray maps. This basic principle has been extended to determine large-scale Galactic variations of $X$ and $\varepsilon_{\gamma}$ (Section 2.3).

In the remainder of this section, the main observational results are presented. Further discussion on the interpretation of the findings is given in Sections 3 and 4.

\subsection{Gamma Rays From the Galactic Disk}

Ever since the first firm detection of celestial $\gamma$ rays by the $O S O-3$ experiment (137), but particularly since the $S A S-2$ observations have become available (83), it has been clear that the $\gamma$-ray sky is dominated by emission from the Galactic disk. The total luminosity of the Galactic disk above $100 \mathrm{MeV}$ is $(1-2) \times 10^{39} \mathrm{erg} \mathrm{s}^{-1}$, of which $\sim 50 \%$ originates beyond the solar circle. Early studies already showed that most of the radiation could be understood, at least qualitatively, in terms of the diffuse processes described above (e.g. 22, 133, 226). Because of the limited angular resolution of $S A S-2$ and $C O S$ - $B$, however, $\gamma$-ray emission from starlike objects might be hidden and would appear part of the diffuse emission to these experiments. Although there were no firm predictions, this possibility had 
to be considered very seriously after the first $C O S-B$ observations showed evidence for several point sources (114) in addition to the few bright ones that could be seen by $S A S-2$ [the Crab $(134,243)$ and Vela $(241,244)$ pulsars, the puzzling source "Geminga" in the Galactic anticenter (243), and Cyg X-3 (142) - the latter is under debate $(89,111)]$. The second COS$B$ catalog $(109,239)$ contains 19 such sources at low Galactic latitudes, none of which could be unambiguously identified. These point sources were defined as significant peaks in the $\gamma$-ray intensity distribution $\left(E_{\gamma}>\right.$ $100 \mathrm{MeV}$ ) with a shape that is consistent with the $C O S-B$ point-spread function. Owing to the poor angular resolution, however, it cannot be excluded that these sources are actually extended; most of the unidentified sources might have an angular diameter up to $\sim 2^{\circ}$.

In the meantime, correlation studies of the observed $\gamma$-ray emission and gas tracers (H I and CO observations) have shown with increasing confidence that most of the $\gamma$-ray emission is probably of diffuse origin. The observed and expected intensity distributions show generally good agreement $(40,147,232)$, and even several unidentified $\gamma$-ray sources in the $C O S-B$ catalog can be attributed to peaks in the gas column density distribution $(170,193,194,202)$. Some other unidentified $\gamma$-ray sources can probably be explained by CR-irradiated clouds as well, if one allows for an enhanced CR density inside the cloud $(116,158,159)$. On the other hand, evidence for some new sources is found when the expected diffuse $\gamma$-ray emission is subtracted from the observed emission. This point is discussed in Section 5.

The main objective of $\gamma$-ray studies of the Galactic disk has usually been to determine the CR distribution in the Galaxy and thereby to extract information on the origin of cosmic rays. This is feasible if independent information is available on the distribution of the target gas particles with which the cosmic rays interact. Early studies suffered in this respect from uncertainties in the molecular-hydrogen distribution in the Galaxy. The atomic-hydrogen distribution was well mapped by $\mathrm{H} \mathrm{I}$ 21-cm line observations, although there were (and still are) some uncertainties due to optical depth effects (e.g. 48). The sky coverage of the observations of the $\mathrm{CO}$ molecule, which is probably the best tracer of the large-scale distribution of $\mathrm{H}_{2}$, was limited to a very narrow band along the Galactic plane. Furthermore, the relation between measured CO intensity and corresponding $\mathrm{H}_{2}$ column density was uncertain. The small latitude extent of the $\mathrm{CO}$ surveys was inadequate for a direct comparison with the $\gamma$-ray observations because of the low angular resolution of the latter. The situation has improved significantly with the availability of the large-scale CO surveys from the Columbia $1.2-\mathrm{m}$ telescopes in New York City and on Cerro Tololo in Chile - the combined survey covers the entire Milky 
Way up to $|b| \approx 7-10^{\circ}$, with several large extensions to higher latitudes (68).

The importance of $\mathrm{H}_{2}$ for the interpretation of the $\gamma$-ray data was, however, certainly realized in early work as well. The $\mathrm{H} \mathrm{I}$ data and the sparse $\mathrm{CO}$ observations (or other information on the $\mathrm{H}_{2}$ distribution) were generally converted into a model of the gas distribution in the Galaxy, although the $\mathrm{CO}-$ to- $\mathrm{H}_{2}$ conversion factor was an uncertain parameter in the modeling. For the second and third Galactic quadrants, the $\mathbf{H}_{2}$ contribution to the gas column densities has long been known to be small on average, so the lack of a complete CO survey did not seriously hamper $\gamma$-ray studies of the outer Galaxy. The $S A S-2$ team generally constructed a spiral-arm model of the gas distribution; based on the assumption that the CR density is proportional to the matter density on the scale of spiral arms, agreement between the predicted and observed $\gamma$-ray intensities was obtained $(22,23,86,100,131,132)$. Paul et al. (187) reached a similar conclusion; they followed a somewhat different approach, however, taking into account the observed nonthermal radio emission of the Galaxy. From follow-up work, Cesarsky et al. (57) concluded that a radial CR density gradient may exist, in addition to CR-matter coupling on the scale of spiral arms (but cosmic rays are assumed to see only half of the molecular gas in this modeling). Fuchs et al. (91) considered CR-matter coupling on larger scales, using a hydrostatic equilibrium model for the ISM. Others did not make a priori assumptions on the proportionality between CR and gas density. The Galacto-centric distribution of $\gamma$-rays was determined from a geometrical unfolding technique applied to the inner-Galaxy $\gamma$-ray data; a comparison with estimates of the radial gas distribution showed that the $\gamma$-ray emissivity $\varepsilon_{\gamma}$ increases toward the inner parts of the Galaxy. The latter approach was generally followed by Stecker and coworkers (99, $224,226)$ and Wolfendale and coworkers $(122,157)$.

The new Columbia CO surveys enabled use of a third method to derive information on the Galactic CR distribution from the $\gamma$-ray data, and this method was applied by the COS-B group. It is an extension of the basic principle described at the end of Section 2.2, namely a multivariate correlation analysis of $\gamma$-ray sky maps with $\mathrm{H}$ I and $\mathrm{CO}$ maps for selected Galacto-centric distance intervals (typically a few kiloparsecs wide). The distance information needed to construct the $\mathrm{HI}$ and $\mathrm{CO}$ maps was derived from the $\mathrm{H} \mathrm{I}$ and $\mathrm{CO}$ line velocities, together with an adopted Galactic rotation curve. The method has the advantage that it takes into account all spatial structures in the data, enabling not only a study of the Galactic CR distribution but also of the $N\left(\mathrm{H}_{2}\right)$-to- $W_{\mathrm{CO}}$ ratio $X$. Basically, this method consists of fitting the observed $\gamma$-ray intensity distributions by the relation $I_{\gamma}=\Sigma \varepsilon_{\gamma}\left(R_{i}\right)\left\{N(\mathrm{HI})_{i}+2 Y W_{\mathrm{CO}, i}\right\}$, where the sum is over the rings 
$i$, and $\varepsilon_{\gamma}\left(R_{i}\right)$ and $Y$ are the free parameters (which were determined by a maximum-likelihood procedure). The fitted model included other components, such as an estimate of the IC contribution, the intense $\gamma$-ray point sources, and an isotropic $\gamma$-ray background. The above relation contains a parameter $Y$ (instead of $X$ ) to remind us of the fact that the CR density inside molecular clouds may differ from that in the ambient medium: $Y=X \cdot \varepsilon_{\gamma, \mathrm{H}_{2}} / \varepsilon_{\gamma, \mathrm{HI}}$, so if CR particles are not excluded from, or concentrated in, molecular clouds, then $Y$ equals $X$ independent of $\gamma$-ray energy. Also, the $Y W_{\mathrm{CO}, i}$ term covers to some extent possible arm/interarm emissivity contrasts because $\mathrm{CO}$ is at least in part a tracer of spiral structure. A first (less complete) study by Lebrun et al. (147) showed that the angular resolution and counting statistics of $C O S-B$ are indeed sufficiently good to put a meaningful $\gamma$-ray constraint on $X$. Bloemen et al. (40) and Strong et al. (232) - the former concentrating mainly on the first and second Galactic quadrants and the latter studying the entire Milky Wayextended this work and derived both $X$ and the Galacto-centric emissivity distribution $\varepsilon_{\gamma}(R)$ for three $\gamma$-ray energy intervals $(70-150 \mathrm{MeV}, 150-300$ $\mathrm{MeV}$, and $300 \mathrm{MeV}-5 \mathrm{GeV}$ - these intervals were chosen, among other reasons, because they have approximately equal counting statistics). Longitude distributions of the observed $\gamma$-ray emission and the fitted model are presented in Figure 3. Figure 4 shows the model sky map for the 100 $\mathrm{MeV}-6 \mathrm{GeV}$ range, based on the fit parameter values derived by Strong et al. [although these were obtained for a more limited latitude range $\left.\left(|b|<10^{\circ}\right)\right]$ and the $\mathrm{H}$ I and $\mathrm{CO}$ surveys used in that work. The map can be compared with the $C O S-B$ map shown in Figure 1. (Note, however, that the model map is not convolved with the $C O S-B$ point-spread function.)

The resulting distributions of $\varepsilon_{y}(R)$ (Figure 5) show only a weak Galactocentric gradient. The same result was obtained from a similar analysis (35, 36) restricted to the outer Galaxy (second and third quadrants), for which it is sufficient to use only $\mathrm{H} \mathrm{I}$ observations as a gas tracer. In fact, for the high-energy range no significant decrease with Galacto-centric radius was found at all beyond the solar circle - although the radial distribution from the recent work described above is the same within uncertainties, it does show a weak gradient, as can be seen in Figure 5. Possible energy dependencies are discussed below in detail. These findings seemed surprising, particularly for the outer Galaxy, because analyses of the $S A S-2$ data had shown evidence for a strong gradient; a steep emissivity decrease with increasing distance beyond the solar circle was first found by Dodds et al. (71) and confirmed by, e.g., Cesarsky et al. (57) and Higdon (115). Later studies of the $S A S-2$ data $(2,35,238)$, however, showed much less evidence for such a strong gradient $\left(E_{\gamma}>100 \mathrm{MeV}\right)$. It turned out that this discrepancy can be attributed to an improvement in the calibration of the 

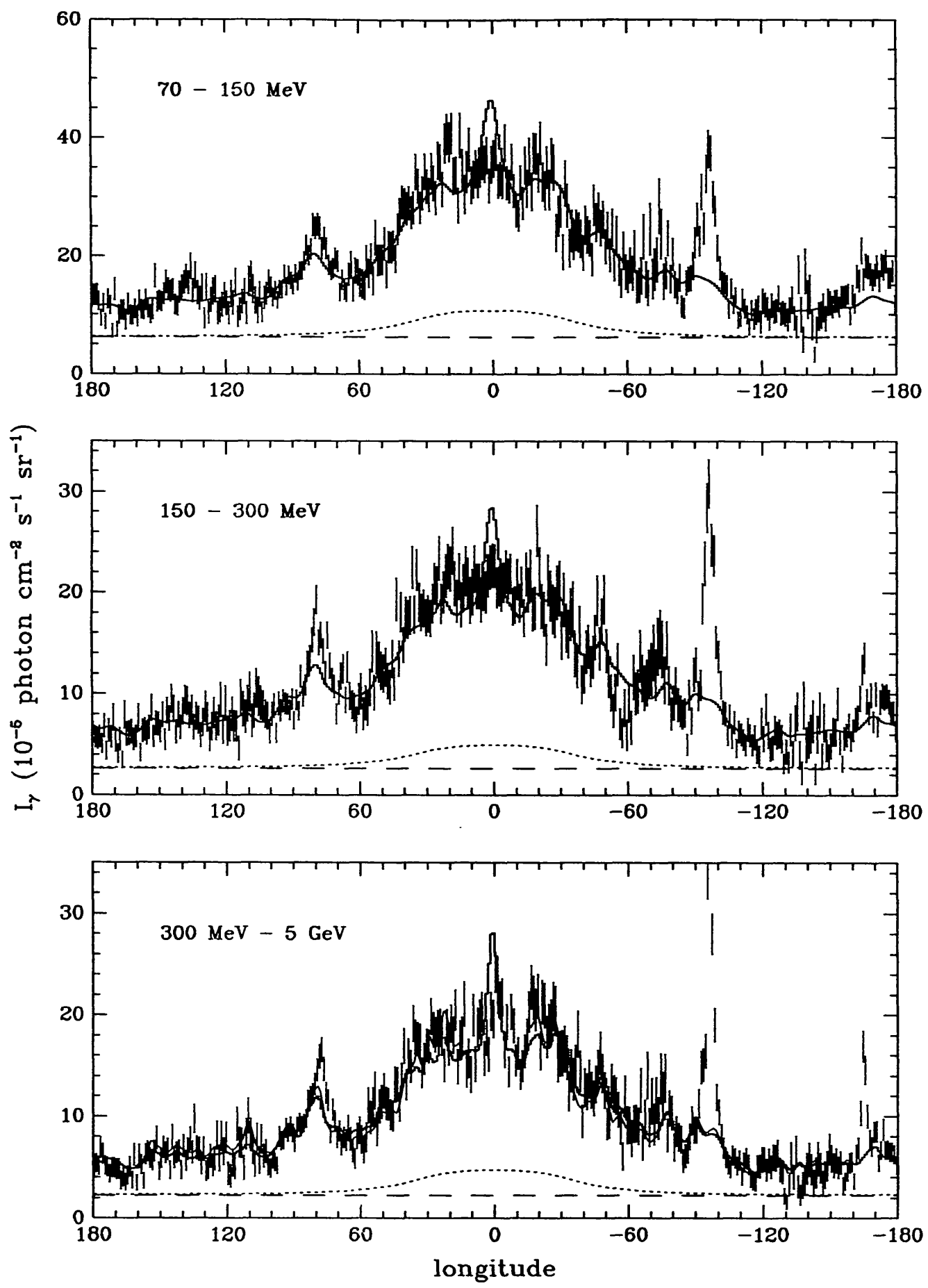

Figure 3 Longitude distributions of the observed ( $\pm 1 \sigma$ error bars) and modeled (histogram) $\gamma$-ray intensity, averaged over $|b|<5^{\circ}$ (232). The dotted line indicates the individual contribution in the model from inverse-Compton emission. The isotropic background is indicated by the dashed line. The specific model shown here has an energy-independent shape of radial emissivity distributions (circles in Figure 5) but appears almost identical in this presentation for an energy-dependent shape (black dots in Figure 5). The thin full line in the figure for the $300 \mathrm{MeV}-5 \mathrm{GeV}$ range indicates the prediction from a model with CR-matter coupling (172), discussed in Section 4.4. 

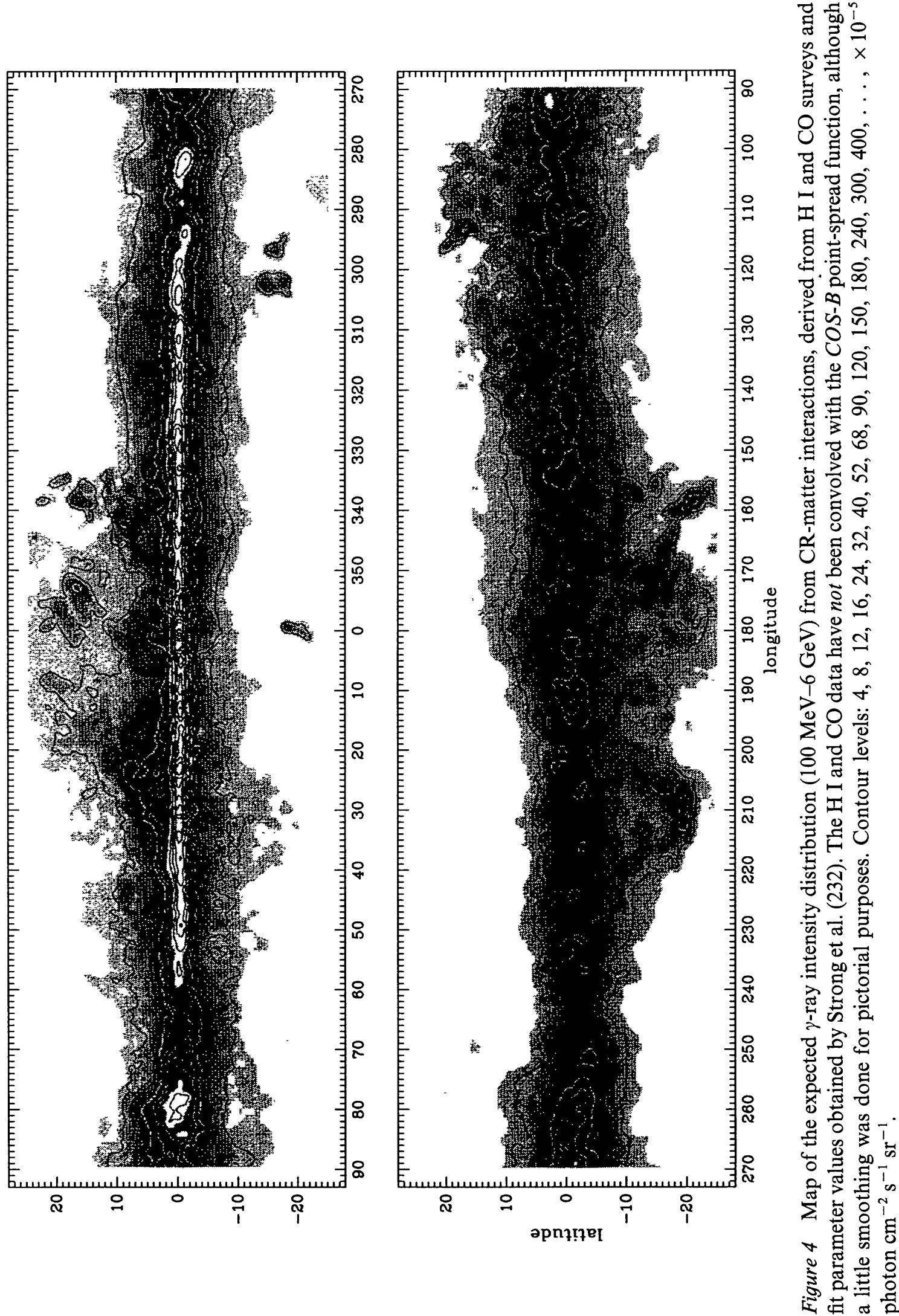


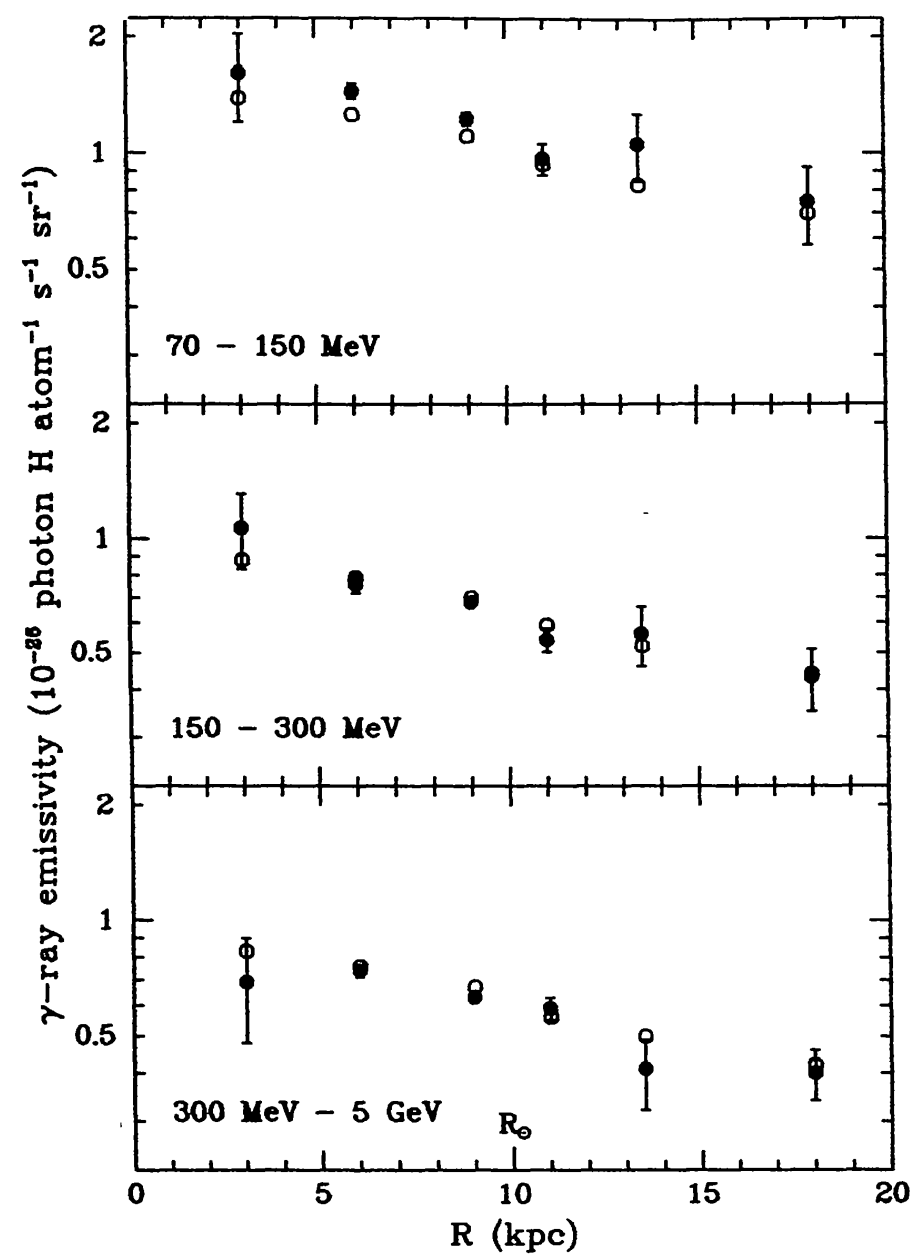

Figure 5 Galacto-centric distribution of the $\gamma$-ray emissivity obtained from a correlation analysis of $\mathrm{H} \mathrm{I}, \mathrm{CO}$, and $\gamma$-ray data (232). (Circles) Shape of the radial distribution is adopted to be independent of $\gamma$-ray energy - the observed large-scale spectral variation along the Milky Way is attributed to an energy dependence of the parameter $Y$ (see Section 2.3.1). (Black dots) Vice versa: $Y$ adopted to be energy independent-spectral variation attributed to an energy dependence of the shape of the radial distribution. Both sets of emissivity distributions lead to an equally good fit of the data; early statistical tests (40) of a subset of the data used to construct this figure preferred the energy-dependent shape, but recent ones (232) prefer the energy-independent shape.

$S A S-2$ data around 1978 [C. Fichtel, personal communication; see Figure 4 in (34)]. As a consequence, the final outer-Galaxy $\gamma$-ray intensities $(>100$ $\mathrm{MeV}$ ) released by the $S A S-2$ group in 1978 (85) are about a factor of two higher than those presented in 1975 (83), which were used in the studies showing the steep gradient.

The Durham group of Wolfendale and collaborators has advocated for many years the presence of a strong gradient. This was to a large extent based on the early $S A S-2$ data, but several of their recent papers $(15,16$, 
$166,240,246,255)$ suggest that there is still no real consensus on the gradient issue, i.e. that in their opinion the emissivity gradient is not as weak as claimed by the $C O S-B$ group. Their present best estimate of the radial emissivity distributions [first presented by Bhat et al. (15) and shown in most of the other papers] was, however, actually taken from Bloemen et al. (40), with the exception that they increased the emissivity values for the smallest radius by $25 \%$, as described by Bhat et al. The point of debate, therefore, appears to be rather minor.

Although most of the recent $\gamma$-ray work on the CR distribution has concentrated on radial gradients, there is still the possibility that this is not an adequate description of the CR distribution in the Galaxy. It was already noted above that the $S A S-2$ group generally assumed proportionality between the CR density and gas density on the scale of spiral arms, which led to a good description of the $\gamma$-ray data. Melisse \& Bloemen (172) have recently reconsidered this possibility, using the $C O S-B$ data and the same $\mathrm{H} \mathrm{I}$ and $\mathrm{CO}$ surveys used for the gradient studies. Their conclusion is that the option of CR-matter coupling is still viable. Details are given in Section 4.4 .

2.3.1 SPECTRAL INFORMATION There is evidence for a large-scale variation of the $\gamma$-ray spectrum along the Milky Way. The first clear indication of this effect came from the COS-B work of Mayer-Hasselwander (167). His longitude distribution $\left(|b|<10^{\circ}\right)$ of the ratio between the intensity in the energy range $70-150 \mathrm{MeV}$ and that in the range $150 \mathrm{MeV}-5 \mathrm{GeV}$ indicates a softer $\gamma$-ray intensity spectrum toward the inner Galaxy than for the remainder of the disk. This result is obviously sensitive to background corrections; in earlier $C O S$ - $B$ work (169), for instance, when the $\gamma$-ray background was less well understood, no spectral variation was found. Since Mayer-Hasselwander did not use the final $\operatorname{COS}-B$ data base, I have repeated his analysis. Another reason for repeating it is the choice of the latitude interval; in order to minimize the influence of $\gamma$ rays that originate in the local ISM and possibly the Galactic halo (see Section 2.4), it is preferable to restrict the interval to $|b|<5^{\circ}$. However, the difference between the angular resolutions of the $70-150 \mathrm{MeV}$ and $150 \mathrm{MeV}-5 \mathrm{GeV}$ energy ranges is not negligible in this case. This difference leads to a stronger broadening of the $\gamma$-ray-emitting Galactic plane for the 70-150 $\mathrm{MeV}$ range, and thus to a larger "spill-over" to $|b|>5^{\circ}$ for this energy range. First-order corrections as a function of longitude can be obtained from the model sky maps of Strong et al. (232) described above [i.e. from comparisons of the model sky maps before and after the convolution with the $C O S-B$ point-spread functions- the method is described in some further detail in (39)]. The correction factors for the spectral ratio turn 
out to be typically $\sim 10 \%$. Figure 6 shows the resulting spectral-ratio distribution and confirms Mayer-Hasselwander's findings.

Indeed, the correlation analyses of Bloemen et al. (40) and Strong et al. (232), discussed above, showed that the interpretation of the $C O S-B$ data requires an energy-dependent model. In these studies, the energy dependence can be attributed to either a higher $Y$ value for low energies than for high energies (which produces a softer spectrum toward the inner Galaxy because most of the molecular gas is located inside the solar circle), or to a steeper $\gamma$-ray emissivity gradient for low energies. These two options can be tested, in principle, because the angular distributions of $\mathbf{H ~ I}$ and $\mathrm{H}_{2}$ are different. In practice, however, such a test is hampered by the degradation of the angular resolution of $C O S-B$ with decreasing energy. This is evident from the results: In the work of Bloemen et al. (40), the likelihood tests favored energy dependence of $\varepsilon_{\gamma}(R)$, whereas the tests by Strong et al. (232) favored energy dependence of $Y$. In both works the two options could not be distinguished at a high level of significance, although the latter is probably to be preferred because Strong et al. used more data. The energy dependence of $Y$ is of course unrelated to $X$, which is by definition energy independent, but it may be related to CR propagation

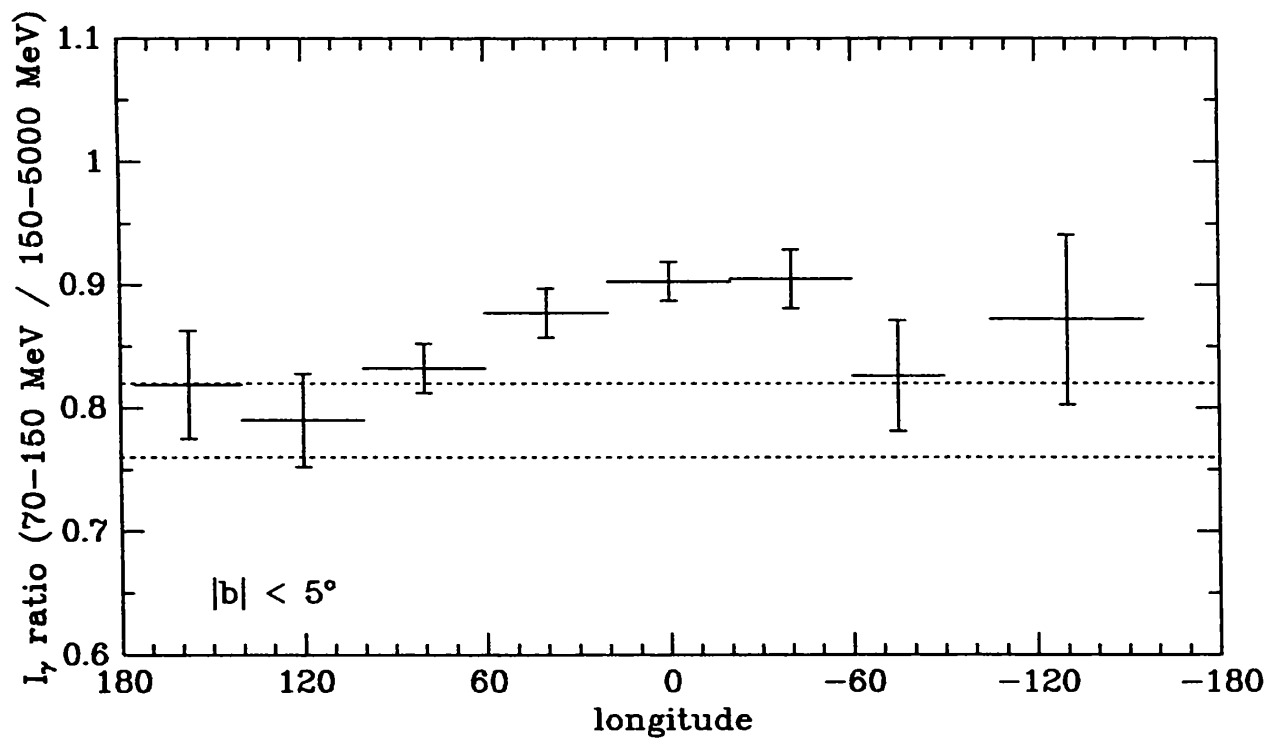

Figure 6 Longitude distribution $\left(|b|<5^{\circ}\right)$ of the spectral ratio between the observed $\gamma$-ray intensity in the $70-150 \mathrm{MeV}$ and $150 \mathrm{MeV}-5 \mathrm{GeV}$ energy ranges, showing a tendency of softer $\gamma$-ray spectra in the inner-Galaxy direction. The correction for differing angular resolutions is described in the text. Regions containing the strong $\gamma$-ray point sources Vela, Crab, and Geminga are excluded. The two dotted lines indicate the range of spectral ratios for typical estimates of local $\gamma$-ray emissivities (see Section 4.2). 


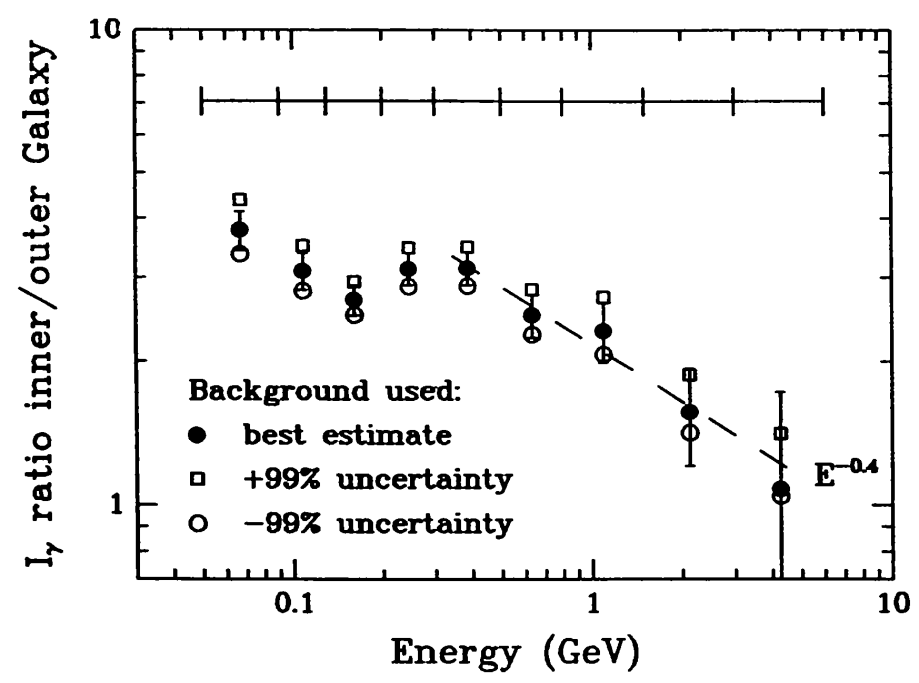

Figure 7 Ratio between the $\gamma$-ray spectra toward the inner $\left(310^{\circ}<l<50^{\circ}\right)$ and the outer Galaxy $\left(90^{\circ}<l<270^{\circ}\right)$, integrated over $|b|<30^{\circ}$ (33). The black dots and error bars correspond to the spectra shown in Figure 2. The squares and circles indicate that uncertainties in the $\gamma$-ray background have only a minor impact.

and production in or near molecular clouds or due to the presence of sources with steep spectra distributed like $\mathrm{CO}$ (Section 4.3). The constraints on $X$ are addressed in Section 3.1; the interpretations with regard to the Galactic CR distribution are discussed in Section 4.3.

In addition to the broad-band spectral difference described above (70$150 \mathrm{MeV}$ versus $150-300 \mathrm{MeV}+300 \mathrm{MeV}-5 \mathrm{GeV}$ ), a large-scale spectral variation exists within the $300 \mathrm{MeV}-5 \mathrm{GeV}$ range. This effect is evident from Figure 7 (33), which shows the ratio between the $\gamma$-ray intensity spectrum in the general direction of the inner Galaxy $\left(310^{\circ}<l<50^{\circ}\right.$; $\left.|b|<30^{\circ}\right)$ and in the direction of the outer Galaxy $\left(90^{\circ}<l<270^{\circ}\right.$; $|b|<30^{\circ}$ ). (The actual spectra are shown in Figure 2.) The outer-Galaxy spectrum above a few hundred $\mathrm{MeV}$ is flatter, with a spectral-index difference of $\sim 0.4$. Later work $(39,204)$ showed, however, that the integration of the emission over the broad latitude range of $|b|<30^{\circ}$ had masked an important effect: The relative flatness of the average outer-Galaxy spectrum results from a gradual spectral hardening with increasing latitude (see Figure 8). Owing to the limited counting statistics, this follow-up work had to be restricted to two energy ranges, namely $300-800 \mathrm{MeV}$ and 800 $\mathrm{MeV}-6 \mathrm{GeV}$. Toward the inner Galaxy, this spectral flattening with latitude is not seen, which is a strong indication that the findings cannot be attributed to systematic uncertainties in the intensity level and spectrum of 

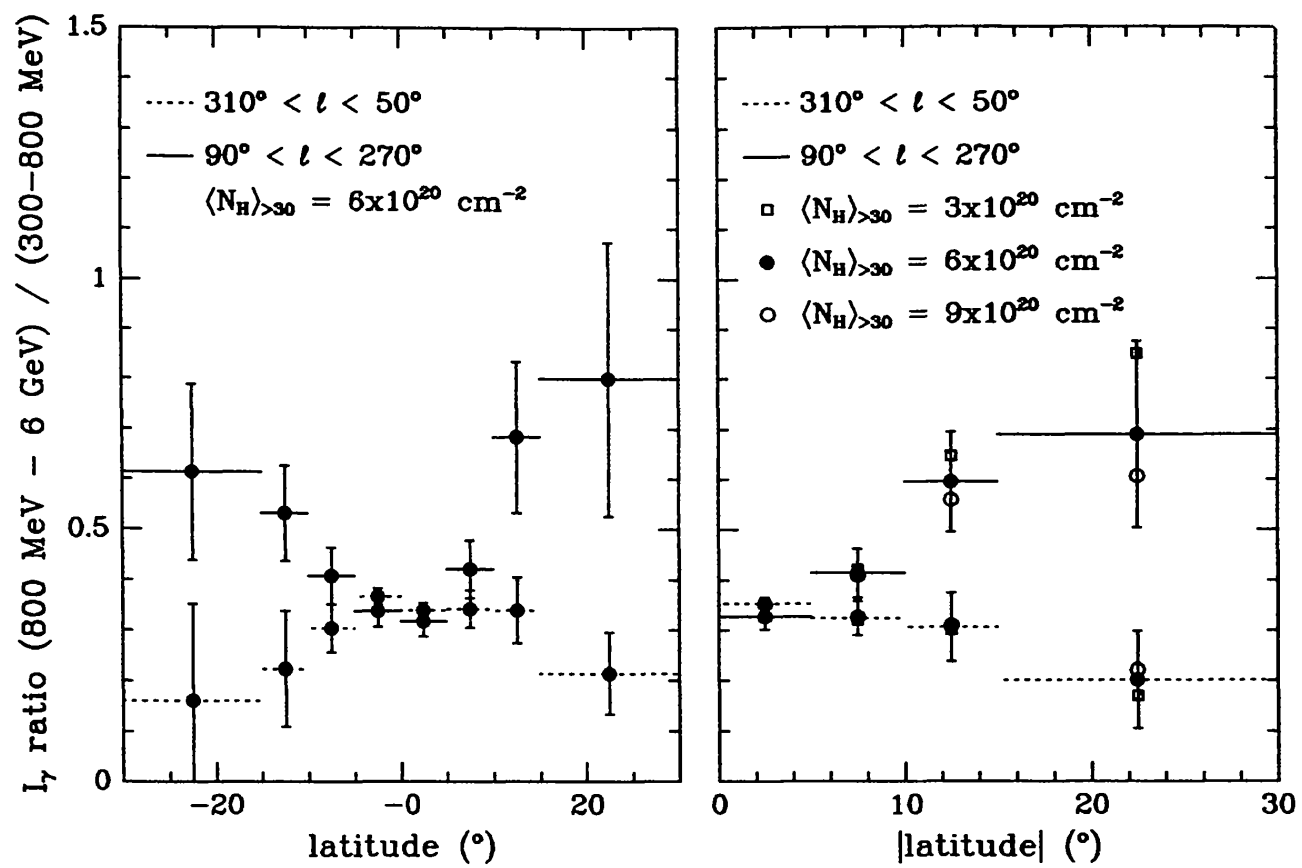

Figure 8 Latitude distribution of the ratio between the intensities for the energy ranges 800 $\mathrm{MeV}-6 \mathrm{GeV}$ and $300-800 \mathrm{MeV}$ in the general directions of the inner and the outer Galaxy (39). (Left) Regions below and above the Galactic plane are shown separately. (Right) Regions on both sides of the plane are combined. The results are shown for different choices of the average gas column density at $|b|>30^{\circ}$, which is related to the background correction made-the figure shows that uncertainties in this correction cannot explain the spectral difference seen toward the inner and outer Galaxy. Details are given in (39).

the isotropic $\gamma$-ray background. The interpretation of this rather surprising result is discussed in Section 4.5.

\subsection{Away From the Galactic Plane: Local ISM and Halo}

Most of the $\gamma$-ray emission observed beyond a few degrees from the Galactic plane is almost certainly of diffuse nature because the $\gamma$-ray point sources detected by $C O S-B$ have a very narrow latitude distribution $\left(\langle|b|\rangle \approx 1.5^{\circ} ; 109,239\right)$, despite the greater detectability away from the intense disk emission. Early analyses of the $S A S-2$ data by Fichtel et al. $(84,88)$ showed that the latitude distribution of the $\gamma$-ray intensity at $|b| \gtrsim 10^{\circ}$ can be described by a two-component model: a component that resembles the latitude distribution of $N(\mathrm{H} \mathrm{I})$, and an isotropic component, possibly of extragalactic origin. In later work, the Lick galaxy counts catalog $(211,212)$ was used to derive total gas column densities; studies of both the $S A S-2$ data $(149,150,237,242)$ and the $C O S-B$ data $(148,228$, $230,231,234)$ showed that the correlation of $\gamma$-ray intensity with total gas 
column density is an improvement over the correlation with $N(\mathrm{H} \mathrm{I})$ alone. ${ }^{1}$ Toward intermediate-latitude regions $\left(10^{\circ} \lesssim|b| \lesssim 20^{\circ}\right)$ in the general direction of the inner Galaxy, however, the observed intensities were found to be significantly larger than expected, particularly at positive latitudes. Both the $S A S-2$ data $(149,150)$ and the $C O S-B$ data $(16,228,230,231$, 234) show this effect. After a recent improvement in the calibration of the galaxy counts, it turned out that the discrepancy is even larger than found previously and that a similar excess is indeed clearly present below the Galactic plane (150). This improvement in the galaxy-counts calibrationwhich reduced the gas column-density estimates for some regions of the sky-resulted from the discovery by Lebrun $(145,146)$ of an observational bias in counting galaxies. The $\gamma$-ray excess is discussed below in further detail.

It is generally assumed that the intermediate-latitude $\gamma$-ray emission originates mainly from the ISM in the solar vicinity. There is strong evidence that this is to a large extent correct, such as the $\gamma$-ray counterparts of Gould's Belt $(83,100,169)$ and possibly Dolidze's Belt $(18,19)$ and the clear detection of $\gamma$ rays from the Orion $(37,53,117,124)$ and Ophiuchus $(113,123)$ molecular-cloud complexes in Gould's Belt. There are indications, however, that a significant fraction of the $\gamma$-ray emission at medium latitudes may not be of local origin, which brings us to the longstanding question of the existence of a $\gamma$-ray halo or "thick disk."

First, for the general direction of the outer Galaxy (second and third Galactic quadrants), it can be estimated from the new Leiden-Green Bank $\mathrm{H}$ I survey $(49,51)$ that $\sim 40 \%$ of the $\mathrm{H}$ I column density at $|b| \approx 15-20^{\circ}$ originates beyond $1 \mathrm{kpc}$ from the Sun $(39,52)$ and thus beyond a few hundred parsecs from the midplane, which is a result of the flaring of the H I disk. This significant contribution of nonlocal gas was less evident from previous medium-latitude $\mathrm{H}$ I surveys $(105,106)$ owing to the limited sensitivity of these observations. Second, the medium-latitude $\gamma$-ray excess toward the inner Galaxy may not be of local origin, as discussed below. A third indication of a nonlocal component in the observed $\gamma$-ray emission at medium latitudes is the spectral flattening above $300 \mathrm{MeV}$ with increasing latitude in the outer-Galaxy direction, as described in Section 2.3. Whatever the precise reason for this flattening may be, the observed symmetry of this effect-it occurs systematically on both sides of the

\footnotetext{
${ }^{1}$ Owing to the highly eccentric orbit of the $C O S-B$ satellite, unlike the near-Earth one chosen for $S A S-2$, the instrumental background of the $C O S-B$ observations is significantly larger than that of the $S A S-2$ observations and dominates the observed emission at high Galactic latitudes. Most $C O S$ - $B$ studies of regions away from the Galactic disk were therefore restricted to medium-latitude regions, up to $|b| \approx 20^{\circ}$.
} 
Galactic plane for the entire second and third Galactic quadrants (39)suggests that it has a large-scale origin. Nevertheless, a local origin cannot be excluded (204). This point is taken up again in Section 4.5.

2.4.1 THE MEDIUM-LATITUDE EXCESS The discrepancy between the observed $\gamma$-ray emission at medium latitudes in the general direction of the inner Galaxy and the expectation from galaxy-counts data is confirmed if one uses $\mathrm{H} \mathrm{I}$ and $\mathrm{CO}$ observations as gas tracers instead of galaxy counts. This was recently shown from a study restricted to the $300 \mathrm{MeV}-5 \mathrm{GeV}$ range (39), but the same holds for lower energies, which is illustrated in Figures 9 and 10. This alternative approach is attractive because it enables usage of only one model for both low- and medium-latitude regions. The $\gamma$-ray expectation shown in Figure 9 is the extension of the empirical model of the disk emission presented by Strong et al. (232), as discussed in Section 2.3; this extension is based on the fit parameter values obtained for $|b|<10^{\circ}$ (i.e. the $\gamma$-ray emissivity values for different Galacto-centric annuli, the $\mathrm{CO}-$ to- $\mathrm{H}_{2}$ conversion factor, and the background levels). The medium-latitude region is certainly not properly covered by the Columbia CO surveys, but this incompleteness cannot explain the excess (39). The longitude range from $l \approx 330^{\circ}$ to $l \approx 40^{\circ}$, for instance, is almost completely mapped at positive latitudes up to $b \approx 20^{\circ}$.

The spectrum of the excess is remarkably soft compared with the emission from the Galactic disk. This was noted by Lebrun \& Paul (150) and can also be seen in the work of Bhat et al. (14), both using $S A S-2$ data. Figures 9 and 10 suggest the same for the $C O S-B$ data, which can be illustrated as follows. Integrating the intensities in Figure 10 over the first and fourth quadrants gives excess fluxes above the Galactic plane $\left(5^{\circ}<b<20^{\circ}\right)$ of $25.3 \pm 1.1,7.8 \pm 0.7$, and $7.0 \pm 0.610^{-6}$ photon $\mathrm{cm}^{-2} \mathrm{~s}^{-1}$ (70-150 MeV, $150-300 \mathrm{MeV}$, and $300 \mathrm{MeV}-5 \mathrm{GeV}$, respectively) and $25.1 \pm 1.2,7.2 \pm 0.8$, and $5.2 \pm 0.610^{-6}$ photon $\mathrm{cm}^{-2} \mathrm{~s}^{-1}$ below the plane $\left(-20^{\circ}<b<-5^{\circ}\right)$. Combining the two regions, one obtains flux ratios of 4.1:1.2:1 (low: medium: high energies). These values can, for instance, be compared with emissivity ratios derived for the solar vicinity, which are typically $1.6: 1.1: 1(40,228,232$; see Section 4.2$)$. The latter values are in good agreement with the expectation from the sum of $\pi^{\circ}$-decay and bremsstrahlung emission for the local electron/proton ratio; using the average of the spectra presented in Figure 2, one obtains 1.63:1.03:1. The spectral softness of the excess is evident. Figure 10 suggests that the excess for the 70-150 MeV interval extends over a wider longitude range than the excess at higher energies. If the zero levels for the three energy ranges are shifted such that optimum fits are obtained for the second and third quadrants (although there is a priori no reason for such a shift), then the 

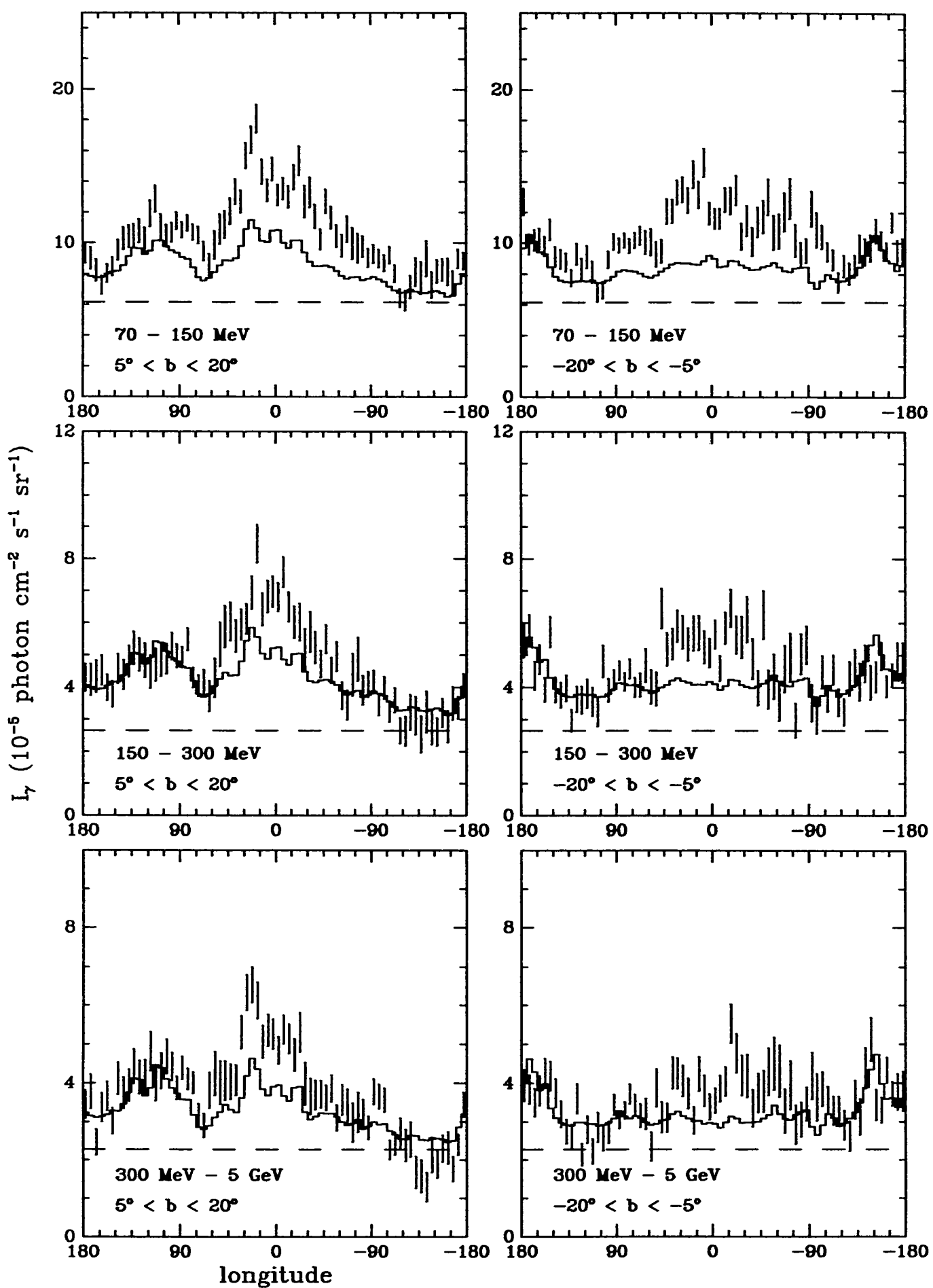

Figure 9 Longitude distributions of the observed ( $\pm 1 \sigma$ error bars) and modeled (histogram) $\gamma$-ray intensities at intermediate latitudes. (Left) Above the Galactic plane, $5^{\circ}<b<20^{\circ}$. (Right) Below the plane, $-20^{\circ}<b<-5^{\circ}$. The model predictions include only CR interactions with atomic and molecular gas and were derived from an extension of the model of the Galactic disk emission presented by Strong et al. (232), which is shown in Figure 3. The dashed line indicates the isotropic background level, also from (232). 


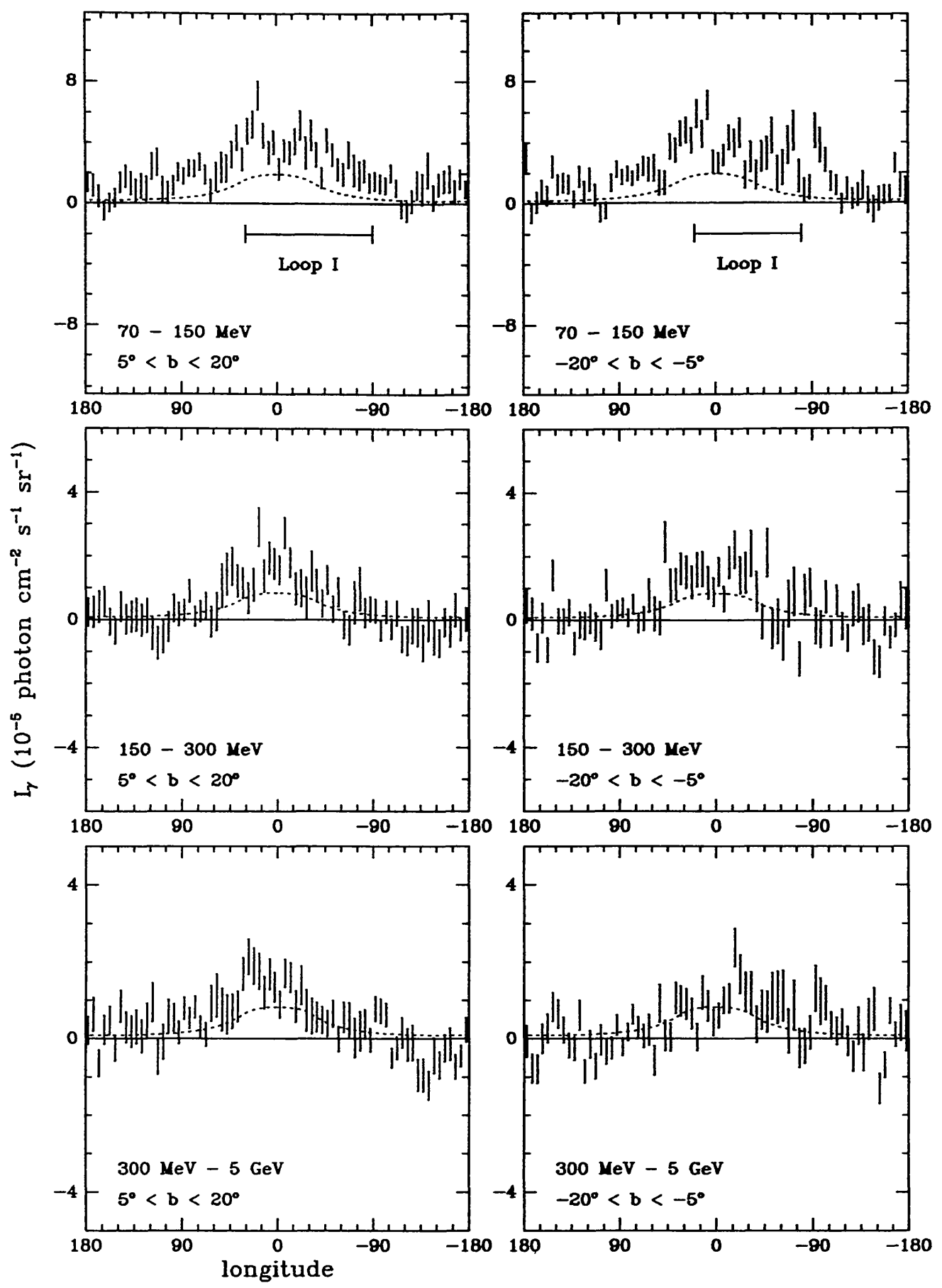

Figure 10 Longitude distributions of the difference between the observed and modeled $\gamma$ ray intensities at intermediate latitudes, as shown in Figure 9. (Left) Above the Galactic plane, $5^{\circ}<b<20^{\circ}$. (Right) Below the plane, $-20<b<-5^{\circ}$. The dotted line shows the prediction from the IC model used by Strong et al. (232). In the upper two figures, the horizontal bars centered on $l \approx 330^{\circ}$ indicate the extent of the Loop I region. 
excess fluxes for the first and fourth quadrants are 16.2, 8.4, and $6.610^{-6}$ photon $\mathrm{cm}^{-2} \mathrm{~s}^{-1}$ above the plane and 16.0, 7.8, and $4.910^{-6}$ photon $\mathrm{cm}^{-2} \mathrm{~s}^{-1}$ below the plane. Combining the two regions gives flux ratios of $2.8: 1.4: 1$; the softness is less pronounced but still very clear.

Several possibilities have been put forward to explain the $\gamma$-ray excess:

1. Some authors $(228,234)$ have attributed a significant fraction of the excess to IC emission. Figure 10 shows that the prediction from the IC model used by Strong et al. (232) explains about half of the excess intensities - most others estimated even lower IC intensities at medium latitudes. On the other hand, there appears to be enough uncertainty in the high-energy electron spectrum and distribution to attribute the entire excess to IC emission (38). In addition, an IC interpretation can account for the soft spectrum. For an $E^{-3}$ electron spectrum (as used in Figure 10), which leads to approximately an $E^{-2}$ IC $\gamma$-ray spectrum (Section 2.1), the IC emissivity ratios are $2.4: 1.1: 1$. For an $E^{-3.4}$ spectrum, the ratios are $3.6: 1.3: 1$.

2. Others $(14,150)$ noted that the excess is possible evidence for an enhanced CR density inside Loop I, which has a radius of $\sim 60^{\circ}$ centered on $l \approx 330^{\circ}, b \approx 18^{\circ}(11)$. Figure 10 suggests, however, that the angular extent of the excess is larger than that of Loop I, particularly at low energies, but it remains a very attractive explanation. The Durham group [Bhat et al. (14), with a recent update given by Van der Walt \& Wolfendale (246)] has considered this possibility in detail. They derive an excess $\gamma$-ray intensity for Loop I of $4.9 \times 10^{-6}$ photon $\mathrm{cm}^{-2} \mathrm{~s}^{-1} \mathrm{sr}^{-1}$ above $100 \mathrm{MeV}$, which agrees with the predicted value of $7 \times 10^{-6}$ as given by Blandford \& Cowie (29). However, the intensity of the excess in Figure 10 is on average almost an order of magnitude larger, namely $\sim 4.5 \times 10^{-5}$ photon $\mathrm{cm}^{-2} \mathrm{~s}^{-1} \mathrm{sr}^{-1}(>100 \mathrm{MeV})$; the Durham group attributed about $90 \%$ of the total excess emission to a local CR gradient.

Rogers \& Wolfendale (205) have made the interesting claim that also Loop III and the Vela supernova remnant are visible in the COS-B data, and that the observed emission is close to theoretical predictions. The detections are not firm, particularly for Loop III, which is claimed to be detected at an intensity level of $10 \%$ of the instrumental background. Detailed studies of the Vela pulsar by Kanbach et al. (128) and Grenier et al. (97) show no evidence of steady $\gamma$-ray emission from the pulsar environment.

3. Another possible reason for the excess is the omission of ionized gas in the $\gamma$-ray prediction (39). There is evidence, particularly from pulsar dispersion-measure data, for the existence of a widespread ionized medium with a scale height of the order of $1 \mathrm{kpc}$ and a midplane density of $\sim 0.03$ 
$\mathrm{cm}^{-3}(63,139,160)$. The contribution of this gas to the total gas column density near the midplane is only a few percent, but it may be comparable to $N(\mathrm{H} \mathrm{I})$ at medium latitudes and thus sufficient to explain the excess flux. [A uniform layer with density $0.03 \mathrm{~cm}^{-3}$ and a thickness of $2 \mathrm{kpc}$ would provide $\sim 0.5 N(\mathrm{H} \mathrm{I})$ at $|b| \approx 15^{\circ}$.] There is not much information on the Galacto-centric distribution of this medium. Obviously, in order to explain the $\gamma$-ray excess this gas should not extend far beyond the solar circle; such a scenario seems plausible because the gas is probably ionized by $O$ stars (164), which are mainly concentrated inside the solar circle. Unless the CR electron-proton ratio is relatively high in this ionized medium (i.e. the bremsstrahlung contribution is relatively high), the spectral softness is hard to understand. The absence of screening in the bremsstrahlung process helps (Secion 2.1) but seems insufficient. It is possible that the ionized gas did not show up in the galaxy counts because of the relatively high destruction rates of interstellar grains in this medium due to the high shock frequencies that can be expected here $(74,75,171)$.

It is not clear whether this soft $\gamma$-ray excess is physically related to the hard $\gamma$-ray spectra (above $300 \mathrm{MeV}$ ) found at medium latitudes in the outer-Galaxy direction. The counting statistics at these high energies are not good enough to investigate whether the intense emission above $\sim 800$ $\mathrm{MeV}$ correlates with the gas distribution or whether it is actually an excess.

\section{RELEVANCE TO STUDIES OF MOLECULAR GAS}

\subsection{The $\mathrm{CO}-\mathrm{H}_{2}$ Calibration}

There has been considerable debate on the use of CO $(J=1 \rightarrow 0)$ observations as a tracer of molecular gas [see, e.g., the discussions by Lequeux (151), Liszt (156), Dickman et al. (70), Verter (247), and Maloney \& Black (162)]. It is not evident that this CO transition can be used at all to obtain $\mathrm{H}_{2}$ column densities because it is optically thick for most molecular clouds. Apart from the $\gamma$-ray evidence discussed below, probably the main empirical justification for using velocity-integrated $\mathrm{CO}$ emission $\left(W_{\mathrm{CO}}\right)$ as a tracer of cloud masses is that the line width has been observed to increase with cloud size $(67,143,174,207,220)$. For the Galactic cloud ensemble, $W_{\text {CO }}$ may then trace $N\left(\mathrm{H}_{2}\right)$ because shadowing of individual clouds is rather unimportant as a result of differential Galactic rotation. Although the $N\left(\mathrm{H}_{2}\right)-W_{\mathrm{CO}}$ conversion factor $X$ may vary from cloud to cloud owing to temperature and abundance variations [see, for instance, the discussion by Maloney \& Black (162)], a measurement of an average value is useful for the determination of $\mathrm{H}_{2}$ masses on a Galactic scale. Gamma-ray obser- 
vations have this potential and provide some insight in a possible largescale (radial) variation of $X$ throughout the Galaxy. Such a gradient may exist because abundance gradients have been found (e.g. 154, 183), although $X$ may not be a strong function of $\mathrm{CO}$ abundance (141, 162, 220 ), and a temperature gradient may be present.

It was already illustrated in Sections 2.2 and 2.3 how $\gamma$-ray observations provide a way of determining $X$. Although this method has the virtue that it does not require any assumptions on excitation, abundance, optical depth, or virial equilibrium, there are two potential problems - namely, (a) the possible existence of unresolved $\gamma$-ray point sources (although their contribution seems small; see Section 2.3), and (b) the possibility that the $\mathrm{CR}$ density in molecular clouds may differ from that in the ambient medium. With regard to $(a)$, if a population of unresolved (genuine) $\gamma$-ray point sources exists, with an angular distribution similar to that of $\mathrm{CO}$, then $X$ is overestimated (i.e. in the terminology of the previous section, $X<Y$ ). With regard to point $(b)$, there are the possibilities of incomplete CR penetration of dense clouds and, alternatively, CR production/ concentration in molecular clouds. Theoretical work indicates that only low-energy CR protons [estimates range from $E_{\mathrm{p}} \lesssim 300 \mathrm{MeV}$ (218) to $\lesssim 50 \mathrm{MeV}$ (59)], which are irrelevant for the production of high-energy $\gamma$ rays, fail to penetrate a dense cloud completely. The alternative, an enhanced CR density in clouds (particularly CR electrons), cannot be excluded (see Section 4.3). So in this case, again, $X<Y$.

Two main groups have used the $C O S-B$ observations to calibrate the $\mathrm{CO}-\mathrm{H}_{2}$ relationship, namely the Durham group of Wolfendale and coworkers, and the $C O S-B$ Collaboration together with the Columbia $\mathrm{CO}$ group. The methods of analysis are different and are discussed here separately. In this discussion " $Y$ " is reserved for the parameter actually determined from the $\gamma$-ray data; " $X$ " is used when either the $Y$ value determined is claimed to be a good estimate of the real $N\left(\mathrm{H}_{2}\right)$-to- $W_{\mathrm{CO}}$ ratio or some interpretation is involved (such as corrections for CR enhancements or point sources).

3.1.1 APPROACH OF THE COS-B GROUP The applied method has been presented in Section 2.3. In summary, $Y$ and $\gamma$-ray emissivities were determined simultaneously from a correlation analysis of $\mathrm{H} \mathrm{I}, \mathrm{CO}$, and $\gamma$-ray sky maps. A first paper by Lebrun et al. (147) concentrated on the first Galactic quadrant. It was restricted to the energy range $300 \mathrm{MeV}-5 \mathrm{GeV}$ (for this work, a constant $\gamma$-ray emissivity was assumed) and gave $Y \approx 3 \times 10^{20}$ molecule $\mathrm{cm}^{-2}\left(\mathrm{~K} \mathrm{~km} \mathrm{~s}^{-1}\right)^{-1}$. The next work [by Bloemen et al. (40)] dealt mainly with the first and second quadrants, giving $Y=(2.75 \pm 0.35) \times 10^{20}(70 \mathrm{MeV}-5 \mathrm{GeV})$. A third paper [by Strong et al. 
(232)] dealt with the whole Galaxy, giving $Y=(2.3 \pm 0.3) \times 10^{20}(150$ MeV-5 GeV). The difference between the last two estimates, although statistically insignificant, results mainly from the different $\gamma$-ray energy intervals used; the third paper, in particular, showed evidence for a larger $Y$ value in the low-energy interval $(70-150 \mathrm{MeV})$, namely $\sim 3.3 \times 10^{20}$, as discussed in Section 2.3. For several reasons (232) the $Y$ value obtained for these low energies is the least reliable indicator of $X$, so the authors stated that the $150 \mathrm{MeV}-5 \mathrm{GeV}$ range is to be preferred for estimating $X$. Recently, Melisse \& Bloemen (172) have extended the work of Strong et al. by allowing for a possible CR-matter coupling. They found that such a coupling cannot be excluded by the data (see Section 4.4), but interestingly, approximately the same $Y$ value results.

Fitting a more extended model to the data, Bloemen et al. and Strong et al. found that the ratio $Y(2-8 \mathrm{kpc}) / Y(>8 \mathrm{kpc})$ is not significantly different from unity $(40,232)$. Hence, there is no indication for a significant difference between the average $X$ value for $R=2-8 \mathrm{kpc}$ and that found for $R>8 \mathrm{kpc}$. Variations on smaller scales cannot be excluded, and the Galactic-center region may deviate (see Section 3.3).

For the reasons given above, the best $Y$ estimate of $\sim 2.3 \times 10^{20}$ should strictly be regarded as an upper limit on $X$. Although the $\gamma$-ray data do not provide a stringent lower limit, there are several reasons to believe that $X$ is not much smaller than $2.3 \times 10^{20}$-namely, $(a)$ the good agreement between model and data; $(b)$ the agreement between the resulting $Y$ value and the one obtained from a similar analysis of the Orion region (37), where the source contribution is probably negligible; and $(c)$ the similar result obtained by using the model that allows for CR-matter coupling.

The $Y$ estimates correspond to the Columbia $\mathrm{CO}$ data, so systematic differences between the Columbia data and other surveys should be taken into account if these estimates are applied to other surveys. Furthermore (and probably needless to say), it is important to keep in mind that the best $Y$ estimate of $2.3 \times 10^{20}$ molecule $\mathrm{cm}^{-2}\left(\mathrm{~K} \mathrm{~km} \mathrm{~s}^{-1}\right)^{-1}$ is an average value for the Galactic cloud ensemble and may not be directly applicable to individual clouds and other galaxies.

3.1.2 APPROACH OF THE DURHAM GROUP Bhat et al. (17) have given a comprehensive account of the Durham analyses regarding the $\mathrm{CO}-\mathrm{H}_{2}$ relationship. An update is given by Wolfendale (254). This section is restricted to their $\gamma$-ray studies. With regard to studies of the Galaxy at large, the most important difference between the Durham approach and that of the COS-B group is that the Durham group does not determine simultaneously $\varepsilon_{\gamma}(R)$ and $Y$. They prefer to use independent information on $\varepsilon_{\gamma}(R)$, which is largely dictated by the fact that the Durham group 
does not use the large-scale Columbia survey but rather $\mathrm{CO}$ observations restricted to regions within $\sim 1^{\circ}$ from the Galactic plane. A complete correlation analysis, as done by the $C O S-B$ group, is therefore not feasible. The radial distribution of $\varepsilon_{\gamma}$ used in their work is mainly based on a correlation analysis of $\gamma$-ray data and $\mathrm{H} \mathrm{I}$ data for selected disk regions at $|b| \gtrsim 2.5^{\circ}$ where the $\mathrm{H}_{2}$ contribution to the gas column density was expected to be small (122). This method is necessarily restricted to Galactocentric radii between $\sim 7$ and $\sim 13 \mathrm{kpc}$, but the resulting emissivity distribution was argued to be similar to that of the surface density of supernova remnants, and the latter was therefore taken to be representative of $\varepsilon_{\gamma}(R)$ throughout the Galaxy. Combining this emissivity distribution with radial unfoldings of the $\gamma$-ray, $\mathrm{H} \mathrm{I}$, and $\mathrm{CO}$ data [the latter taken from Sanders et al. (208)], Bhat et al. (13) found $Y \approx 0.7 \times 10^{20}$ molecule $\mathrm{cm}^{-2}\left(\mathrm{~K} \mathrm{~km} \mathrm{~s}^{-1}\right)^{-1}$ at $R \approx 6 \mathrm{kpc}$. Bhat et al. $(13,17)$ claim independent support for this low $X$ value in the inner Galaxy from X-ray absorption, infrared observations, and the virial theorem (update in 161), which led to their best estimate of $X \approx 1.0 \times 10^{20}$ molecule $\mathrm{cm}^{-2}\left(\mathrm{~K} \mathrm{~km} \mathrm{~s}^{-1}\right)^{-1}$ at $R \approx 5-6 \mathrm{kpc}$.

The Durham group has derived an average $X$ value for the solar vicinity from studies of several individual molecular clouds and cloud complexes. The $\gamma$-ray emissivity was mostly adopted to be the mean value of a number of determinations at intermediate latitudes, obtained from correlation analyses of $\gamma$-ray and galaxy-counts data [as summarized in (237)]. Bhat et al. (13) compared LTE-mass estimates of molecular clouds with the fluxes of $\gamma$-ray excesses at or near the positions of these clouds [an update of the work of Issa \& Wolfendale (125)] and found $Y \approx 1.2 \times 10^{20}$. For Orion clouds $\mathrm{A}$ and $\mathrm{B}$, Houston \& Wolfendale (117) found $Y=(1.9 \pm$ $0.5) \times 10^{20}$ from a correlation study of $\gamma$-ray intensity and $W_{\mathrm{CO}}$. Richardson \& Wolfendale $(199,200)$, dealing with the molecular clouds in Taurus, Cepheus, and Orion, have reported that $Y$ shows a falloff with increasing $\gamma$-ray energy, ranging from $\sim 2-2.5 \times 10^{20}$ at $100 \mathrm{MeV}$ to $\sim 1.5 \times 10^{20}$ at $1 \mathrm{GeV}$. The evidence for this effect is at a marginal level of significance, but it is in qualitative agreement with their earlier work on the inner Galaxy (201) and with the relatively high $Y$ value for the 70-150 MeV range found by the $C O S$ - $B$ group. The best Durham estimate of the local $X$ value obtained from this work is $\sim 1.5 \times 10^{20}$ molecule $\mathrm{cm}^{-2}\left(\mathrm{~K} \mathrm{~km} \mathrm{~s}^{-1}\right)^{-1}$.

3.1.3 COMPARISON Although the results from the two groups are not drastically different, there is some discrepancy, at least in the conclusions. Irrespective of the differences, however, it is clear that $\gamma$-ray observations favor rather low $X$ values [other principal determinations are compiled in, e.g., (40)]. The most important discrepancy between the findings of the 
two groups corresponds to the so-called molecular ring at 4-7 kpc from the Galactic center, where most of the molecular gas in the Galaxy is located. The Durham group obtained a significantly smaller $Y$ value here than the $C O S-B$ group. In addition, the Durham group claims that $Y$ (as well as $X$ ) is smaller in the inner Galaxy than in the solar vicinity, whereas the $C O S-B$ group finds no significant evidence for such a radial dependence. The reason for the differing results appears to be twofold: $(a)$ The radial $\gamma$-ray emissivity gradient adopted by the Durham group for this work is steeper than the one derived by the $C O S-B$ group, and $(b)$ the specific CO surface densities used by the Durham group [namely those presented by Sanders et al. (208)] are relatively high in comparison with the Columbia results. With regard to $(a)$, the Durham group assumes an emissivity increase by a factor of $\sim 2.2$ between $R=10$ and $5 \mathrm{kpc}$ (13), whereas the $C O S-B$ group finds an increase of only $\sim 25 \%$ (Figure 5 ). Point $(b)$ is discussed in detail by Bronfman et al. (44), who showed that the high CO surface densities presented by Sanders et al. can be attributed to the following two facts. Firstly, the $W_{\text {CO }}$ values of the survey used by Sanders et al. are simply $20 \%$ higher than those of the Columbia survey. Secondly, there is some debate among CO observers on the radial-unfolding procedure to be applied; using the procedure advocated by Bronfman et al. would lead to an average $\mathrm{CO}$ surface density in the inner Galaxy that is $\sim 40 \%$ lower than that derived by Sanders et al. The method used by the $C O S-B$ group does not depend on such unfolding procedures. Altogether, these effects account for a correction factor of $(2.2 / 1.25) \times$ $1.2 \times 1.4 \approx 3.0$ (although the factor of 1.4 corresponding to the unfolding procedures may, of course, not be applicable). This renders the difference between the derived $Y$ values fully understandable.

\subsection{The Molecular Gas Content of the Galaxy}

The good correlation between the observed $\gamma$-ray intensity distribution of the Milky Way and the expectation from $\mathrm{H}$ I and $\mathrm{CO}$ observations, with a simple linear $N\left(\mathrm{H}_{2}\right)-W_{\mathrm{CO}}$ relationship, indicates that $\mathrm{CO}$ luminosity is a good large-scale mass tracer. The $C O S-B$ group estimated a total $\mathrm{H}_{2}$ mass in the inner Galaxy $(2-10 \mathrm{kpc})$ of $\sim 1.0 \times 10^{9} M_{\odot}(40,232)$, which is, strictly speaking, an upper limit; the Durham group found $\sim 0.6 \times 10^{9}$ $M_{\odot}(13,17)$. For comparison, Henderson et al. (108) derived an H I mass in the inner Galaxy of $\sim 0.9 \times 10^{9} M_{\odot}$, and thus the $\gamma$-ray observations favor rough equality in the $\mathrm{H} \mathrm{I}$ and $\mathrm{H}_{2}$ masses in the inner Galaxy (within a few hundred parsecs from the midplane). The $C O S-B$ mass estimates from the Columbia data have been derived in two different ways, both of which led to approximately the same result - namely, by scaling to the H I mass in the inner Galaxy [for details, see (40)] and by scaling the $\mathrm{H}_{2}$ mass 
derived by Bronfman et al. (44) from the Columbia data with the updated $X$ value. In both cases, the obtained $\mathrm{H}_{2}$ mass is not affected by uncertainties in the absolute $\mathrm{CO}$ and $\gamma$-ray intensity calibrations.

The $\gamma$-ray observations clearly favor the lowest $\mathbf{H}_{2}$ masses obtained in previous studies [see, e.g., the compilation given in (40)]. Bloemen et al. (40) found that the $\mathrm{H}_{2}$ mass even in the $2-8 \mathrm{kpc}$ annulus exceeds the $\mathrm{H} \mathrm{I}$ mass by not more than $\sim 35 \%$. The $\mathrm{H}_{2} / \mathrm{H}$ I mass ratio may be higher or lower at the peak of the molecular ring depending on the actual variation of $X$ within the 2-8 kpc annulus. Outside the solar circle, for $R<15 \mathrm{kpc}$, the $\gamma$-ray results indicate that $M\left(\mathrm{H}_{2}\right)$ is $\sim 20 \%$ of the total gas mass, namely $\sim 2.5 \times 10^{8} M_{\odot}(36,40)$, compared with $M(\mathrm{H} \mathrm{I}) \approx 1.1 \times 10^{9} M_{\odot}$ (108). Altogether, the $\gamma$-ray observations show that the total $\mathbf{H}_{2}$ mass in the Galaxy does not exceed $1.2 \times 10^{9} M_{\odot}$, compared with a total $\mathrm{H}$ I mass of $\sim 4.8 \times 10^{9} M_{\odot}(108)$.

\subsection{The Galactic Center}

The central $400 \mathrm{pc}$ of the Milky Way (here referred to as the Galactic center) contains a very large column density of $\mathrm{CO}$ and other molecules that is about 5-10 times greater than that of a typical line of sight through the entire Galactic disk (e.g. 181, and references therein). This concentration of molecules can be expected to show up as a strong peak in the $\gamma$-ray observations, if $X$ and $\varepsilon_{\gamma}$ near the Galactic center are similar to the values measured for the Galactic disk. (This assumption was made in Figure 3.) Blitz et al. (30) analyzed the $C O S-B$ data and showed that such an excess is lacking, or, more specifically, that the $\gamma$-ray flux $\left(E_{\gamma}>300\right.$ $\mathrm{MeV}$ ) from the Galactic center is nearly an order of magnitude smaller than expected on this basis. Bhat et al. (13) have reached a similar conclusion. The derived $(3 \sigma)$ upper limit of $4 \times 10^{-7}$ photon $\mathrm{cm}^{-2} \mathrm{~s}^{-1}(300$ $\mathrm{MeV}-5 \mathrm{GeV}$ ) on the $\gamma$-ray flux from the Galactic center is lower than the values obtained from earlier studies of the $S A S-2$ and $C O S-B$ data (4, 122), primarily because of the much larger $\mathrm{CO}$ and $\gamma$-ray data bases that are available now. The new result implies that in the Galactic center either the $\gamma$-ray emissivity is anomalously low or $\mathrm{H}_{2}$ is nearly an order of magnitude less abundant than estimates made from $\mathrm{CO}$ observations with an average Galactic $X$ value. The former suggests that the density of CR protons with $\mathrm{GeV}$ energies is anomalously small relative to the local value, or perhaps that these cosmic rays do not efficiently penetrate the molecular clouds. The latter implies that the $3 \sigma$ upper limit to the $\mathbf{H}_{2}$ mass of the Galactic center is only $\sim 6 \times 10^{7} M_{\odot}$, which is at least an order of magnitude smaller than that previously advocated by millimeter-line observers (e.g. 107, 155, 208).

Although it could not be decided from these $\gamma$-ray analyses whether the 
Galactic center $\gamma$-ray deficit is due to a low $\gamma$-ray emissivity or a low $X$ value, there is some circumstantial evidence in favor of a low $X$ value, as discussed by Blitz et al. (30) and Stacy et al. (221). On the other hand, for the highest energies (1-5 GeV) observable with $C O S-B$, Silk \& Bloemen (215) did not find evidence for a $\gamma$-ray deficiency, but this finding needs to be studied in further detail. Since the $\pi^{\circ}$-decay $\gamma$-ray emission at $E_{\gamma}>1$ $\mathrm{GeV}$ originates largely from CR protons with energies $E_{\mathrm{p}} \gtrsim 5 \mathrm{GeV}$ (see, e.g., 69), these results may indicate that the CR spectrum near the Galactic center is "normal" above $\sim 5 \mathrm{GeV}$ but flattens off at lower energies in comparison with the average spectrum in the Galactic disk. A possible explanation is that the Galactic center produces a wind, which tends to exclude low-energy particles and modifies the spectrum of the cosmic rays propagating near the Galactic center, just as the solar modulation affects local cosmic rays. This possibility requires further attention.

\section{RELEVANCE TO STUDIES OF COSMIC RAYS}

\subsection{Some Background}

The CR particle flux measured near Earth consists principally of protons, to which helium nuclei contribute about $10 \%$ and heavier nuclei and electrons (+ positrons) each add less than a few percent. A comprehensive review of the CR composition and its energy dependence is given by Simpson (217). The abundance distribution of cosmic rays is similar to that of the solar system and the local region of the Galaxy, but there are also some significant deviations. The most obvious deviations are the overabundances in cosmic rays of the spallation products lithium, beryllium, boron, and the subiron elements $(20<Z<26)$, which are largely produced by $\mathrm{CR}$ interactions with interstellar matter. These secondary particles play an important role for the understanding of CR propagation and confinement [see, e.g., the review by Cesarsky (55)]. Several other deviations remain after the fragmentation processes in the ISM have been taken into account. These differences give important clues on the origin of cosmic rays. An extensive review is given by Meyer (173).

At least in the solar vicinity, the energy density of cosmic rays is comparable to that of the magnetic field, the interstellar gas (both thermal and macroscopic motions), and the interstellar photon field (all $\sim 1 \mathrm{eV} \mathrm{cm}^{-3}$ ). Ever since the classical work of Parker (184-186), it has been well known that the cosmic rays and magnetic field may be of essential importance for understanding the structure and dynamics of the ISM. The CR energy density can largely be ascribed to protons with energies between about 1 $\mathrm{GeV}$ and a few tens of $\mathrm{GeV}$, which also generate probably the bulk of the observed high-energy $\gamma$ rays at $E_{\gamma} \gtrsim 100 \mathrm{MeV}$ (Section 2.1). Clearly, $\gamma$-ray 
observations trace an interesting part of the $\mathrm{CR}$ spectrum. One important aspect is the unique possibility to study the Galactic distribution of these particles. Furthermore, $\gamma$-ray spectra reflect the CR spectral distribution, which contains information on CR propagation characteristics. At $\gamma$-ray energies below $\sim 200 \mathrm{MeV}$, the observations trace the electron spectrum below $\sim 1 \mathrm{GeV}$, which adds useful information to CR electron studies based on low-frequency radio observations of the diffuse Galactic synchrotron emission and to direct CR electron measurements near Earth.

Shock acceleration $(7,10,27,138)$, i.e. first-order Fermi acceleration in strong shocks induced mainly by supernova remnants, is at present the favored mechanism for the production of cosmic rays with total energies up to $\sim 10^{5} \mathrm{GeV}$ [see, e.g., the review by Axford (6)]. It produces quite naturally a power-law CR spectrum with a spectral index of 2 or somewhat larger, which is consistent with the CR source spectrum inferred from observations, and it can account for the power required to produce the observed CR energy density. Cosmic rays may get reaccelerated when encountering shocks in the ISM (28) or by second-order Fermi acceleration in interstellar turbulence (81); continuous acceleration, occurring solely during propagation, is, however, very hard to reconcile with the observed abundance ratios of secondary and primary particles and with the observed power-law shape of CR spectra $(64,65,77,102)$. Cesarsky (56) has reviewed the state of the art of this topic. Although the nonlinear aspects of shock acceleration are not fully understood yet (e.g. 249), the mechanism is very attractive and the problem of the origin of cosmic rays $\left(\lesssim 10^{5} \mathrm{GeV}\right)$ seems to be shifting towards finding the CR injectors, i.e. the sources that provide the CR material and speed it up to moderate suprathermal energies. On the basis of energetics as well as CR composition, stellar flares and winds are considered to be likely candidates $(3,54,173)$, although others have argued that CR injectors are not needed and that shocks can (or have to) accelerate particles directly out of an interstellar plasma (5, $76,77)$.

There is no conclusive proof of the confinement region of cosmic rays. At least the CR electrons have a Galactic origin, because they cannot survive the Compton losses in the microwave background. It is in principle possible, however, that (part of) the CR proton-nuclear component fills a much larger volume than that of a galaxy, such as a (super)cluster, as advocated by Burbidge $(42,46,47)$. This possibility has frequently been criticized, particularly by Ginzburg and collaborators (e.g. 94, 95). As cosmic rays are closely attached to the interstellar magnetic field lines because of their relatively small gyroradii (for $10-\mathrm{GeV}$ protons in a field of a few $\mu \mathrm{G}$, for instance, $r_{\mathrm{g}} \approx 10^{-6} \mathrm{pc}$ ), their propagation is largely determined by the characteristics of the field. Starting with the work of 
Pikelner (192), Shklovskii (213), and Ginzburg (92) in the early 1950s, many observational and theoretical studies have indicated that the distributions of the magnetic field and cosmic rays extend far beyond the Galactic disk. Direct evidence for the existence of such halos or thick disks follows from low-frequency radio-continuum observations of our Galaxy $(8,12,43,189,190)$ and some edge-on galaxies $(1,9,78,118,119,129$, 130 ), with indirect support from the observed composition and spectra of cosmic rays. Several studies of the polarization of starlight and radio synchrotron emission and of the rotation measures of pulsars and extragalactic radio sources [reviewed by Sofue et al. (219) and Heiles (103)] have shown that the Galactic magnetic field consists of a systematic component, preferentially aligned parallel to the Galactic plane, and an irregular component of comparable strength. Owing to the presence of this tangled component, the CR particles will not only diffuse along the field lines, but also will spread around to other field lines (195). It has been argued that CR propagation can therefore be described in first-order approximation as isotropic diffusion (93). Alternatively, in a so-called dynamical halo model, the magnetic field and CR particles can be convected away from the disk in a Galactic wind $(126,127,136,182)$; cosmic rays might even help to power a wind (121). In addition to the CR density gradients on a Galactic scale, which can be expected from these processes, several scenarios that lead to small-scale gradients have been proposed, such as trapping of cosmic rays near their sources (66), in spiral arms (188), or in tunnels (210). Cesarsky (55) has discussed these transport and confinement models in detail. We see below that $\gamma$ rays give some insight into the role of the diffusion and convection processes and the confinement of cosmic rays.

\subsection{Cosmic Rays in the Solar Vicinity}

Given the $\gamma$-ray emissivity spectrum of the local ISM, some information can be deduced on the local CR electron and proton spectra. This is particularly useful for the CR electrons, because the low energies that can be studied this way ( $50 \mathrm{MeV} \lesssim E_{\mathrm{e}} \lesssim 500 \mathrm{MeV}$ ) can barely be addressed by direct CR measurements (as a result of strong solar modulation for $E_{\mathrm{e}}<1 \mathrm{GeV}$ ) and radio measurements (as a result of strong free-free absorption for frequencies below $\sim 5 \mathrm{MHz}$, corresponding roughly to $E_{\mathrm{e}} \lesssim 300 \mathrm{MeV}$ ). The shape of the electron spectrum between about $1 \mathrm{GeV}$ and $10 \mathrm{GeV}$ is reflected in the spectral shape of radio continuum observations between about $50 \mathrm{MHz}$ and $5 \mathrm{GHz}$ [the directions of the Galactic poles and anticenter are mostly chosen for these analyses (203, 251)] and can be normalized to the directly observed electron spectrum at $\sim 10 \mathrm{GeV}$. 
The importance of $\gamma$-ray observations for the determination of the lowenergy electron spectrum was first discussed in detail by Fichtel et al. (87). The constraints have successively been studied by Strong et al. (238), Strong \& Wolfendale (236), Cesarsky et al. (58), Lebrun \& Paul (149), Lebrun et al. (148), Gualandris \& Strong (98), and Strong (229). It is not possible to discuss here the different approaches, but the basic idea should be clear from Section 2.1-namely, that given an estimate of the $\pi^{\circ}$ decay $\gamma$-ray spectrum (based on CR proton measurements near Earth), the bremsstrahlung $\gamma$-ray spectrum and thus the electron spectrum can be obtained for a given measurement of the local $\gamma$-ray emissivity spectrum. A common conclusion drawn from these studies is that the $\gamma$-ray estimate of the low-energy electron flux at $E_{\mathrm{e}} \simeq 100 \mathrm{MeV}$ lies above plausible extrapolations of the electron spectrum constructed from direct electron measurements and radio observations. In the course of these studies, however, the discrepancy has become smaller:

- The estimate of the low-energy electron flux from radio and direct electron measurements went up. The reason, as discussed by Webber (250), is that an uncertainty by a factor of about two existed in trying to normalize the demodulated electron spectrum and the radio spectrum at $E_{\mathrm{e}} \simeq 10 \mathrm{GeV}$, but the measurements have converged to the previous "high" spectrum.

- The $\gamma$-ray estimate of the electron flux went down. The main reason is that the $\gamma$-ray emissivity value for low energies has decreased; in the $C O S-B$ energy range $70-150 \mathrm{MeV}$, for instance, the values have changed from $\varepsilon_{\gamma} \simeq 1.4 \times 10^{-26}$ photon $(\mathrm{H} \text { atom })^{-1} \mathrm{~s}^{-1} \mathrm{sr}^{-1}(35,231)$ to $(1.0$ $1.1) \times 10^{-26}(40,232,234)$, largely because of the availability of more data and improvements in the analyses.

Table 1 summarizes the present best estimates of local $\gamma$-ray emissivities

Table 1 Local $\gamma$-ray emissivities

\begin{tabular}{ccccccc}
\hline \hline $\begin{array}{c}E \\
(\mathrm{MeV})\end{array}$ & Total $^{\mathrm{a}}$ & Total $^{\mathrm{b}}$ & $\begin{array}{c}\pi^{\mathrm{o}} \text {-decay } \\
{\left[10^{-26} \text { photon }\right.}\end{array}$ & $\begin{array}{c}\pi^{\mathrm{o}} \text {-decay } \text { atom }^{-1} \\
\mathrm{~s}^{-1}\end{array}$ & $\begin{array}{c}\pi^{\mathrm{o}} \text {-decay } \\
\left.\mathrm{Sr}^{-1}\right]\end{array}$ & Bremsstrahlung $^{\mathrm{f}}$ \\
\hline $70-150$ & $1.10 \pm 0.08$ & $1.02 \pm 0.10$ & 0.44 & 0.52 & $0.40 \pm 0.10$ & 0.48 \\
$150-300$ & $0.76 \pm 0.09$ & $0.65 \pm 0.06$ & 0.37 & 0.49 & $0.41 \pm 0.08$ & 0.20 \\
$300-5000$ & $0.68 \pm 0.09$ & $0.62 \pm 0.06$ & 0.45 & 0.49 & $0.56 \pm 0.09$ & 0.10 \\
\hline
\end{tabular}

${ }^{a}$ Measurement: From correlation study at medium latitudes $\left(10^{\circ}<|b|<20^{\circ}\right)(234)$.

${ }^{\mathrm{b}}$ Measurement: From correlation study of the Galactic disk $\left(|b|<10^{\circ}\right)(232)$.

${ }^{\mathrm{c}}$ Prediction: Derived by Stephens \& Badhwar (227) (their B\&S curve).

${ }^{\mathrm{d}}$ Prediction: Derived by Stephens \& Badhwar (227) (their $M_{\mathrm{U}}$ curve).

'Prediction: Derived by Dermer (69).

${ }^{\mathrm{r}}$ Prediction: Derived from electron spectrum given by Webber $\left(250\right.$, his Figure 1) with $E_{\mathrm{e}}^{-2.4}$ extrapolation for $E_{\mathrm{e}}$ $<300 \mathrm{MeV}$. 
from two different studies (near the Galactic plane and at medium latitudes), together with $\pi^{\circ}$-decay and bremsstrahlung predictions. The $\pi^{\circ}$ decay predictions are based on the demodulated proton spectrum; the differences originate from the different demodulation procedures and cross sections used. The bremsstrahlung estimates were derived from the maximum low-energy electron spectrum that Webber (250) considers acceptable. Measurements and predictions are clearly very close now.

\subsection{Radial Gradients}

If cosmic rays are of Galactic origin, then a significant radial gradient of the CR intensity can be expected on a large scale in the Galaxy, simply because more potential CR sources are present in the inner regions. Effective mixing of cosmic rays in the Galaxy (as in a halo diffusion model) may, however, lead to a much weaker falloff for the particle distribution than for the source distribution, so an absence of a strong gradient does not necessarily imply an extragalactic origin. Several studies of the radial CR distribution have been performed in the past, using the $\gamma$-ray emissivity as a measure of the CR intensity. The basic findings were discussed in Section 2.3. It is clear from that discussion that there has been some confusion due to uncertainties in the $\mathrm{H}_{2}$ content of the Galaxy and to differences between recent results and those obtained from early studies of the $S A S-2$ survey, before the release of the final data base. At present, the principal conclusion that can be drawn is that radial emissivity gradients are present over the Galaxy as a whole, for the entire $\gamma$-ray energy range studied, but the gradients are much weaker than found in early work. The radial emissivity distributions obtained from the latest and most robust analysis (232) were shown in Figure 5. Thus, the CR intensity appears to decrease weakly with Galacto-centric radius, although the electron and proton-nuclear components of relevance here may behave somewhat differently, as discussed below.

We saw in Section 2.3 that the emissivity gradient may be stronger for the low-energy band studied (70-150 MeV) than for the two other bands, but there was a question mark. To recall the situation: There is good evidence that the interpretation of the $\gamma$-ray data requires an energydependent model such that the $\gamma$-ray intensity spectrum toward the inner Galaxy is softer (i.e. a surplus of 70-150 MeV $\gamma$ rays) than for the remainder of the disk, suggesting that the $\gamma$-ray emissivity spectrum is softer in the inner regions. However, it is still debatable whether $(a)$ this holds only for the molecular clouds or $(b)$ it is a ubiquitous phenomenon. Also, it cannot be excluded that a concentration of unresolved steep-spectrum $\gamma$-ray point sources in the inner Galaxy is responsible for the soft spectrum. 
(a) ENERGY-INDEPENDENT GRADIENT/SOFT $\gamma$-RAY SPECTRUM FOR MOLECULAR CLOUDS If the gradient is assumed to be energy independent, then it follows that the radial (exponential) scale length for both the electrons and protons is as large as $\sim 15 \mathrm{kpc}$ for $R \gtrsim 4 \mathrm{kpc}(40,232)$. In contrast, the objects that are generally considered to be potential CR sources, injecting the particles and/or accelerating them, such as supernova remnants, earlytype stars, and in fact disk stars in general, all have radial scale lengths of typically $\sim 5 \mathrm{kpc}$ for $R \gtrsim 4 \mathrm{kpc}(135,160,165)$. In order to investigate whether this difference can be understood in a CR diffusion model, Dogiel \& Uryson (73) and Bloemen \& Dogiel (38) have recently numerically modeled the situation with a source distribution resembling that of supernova remnants and different choices for the dimensions of the CR halo. The diffusion coefficient ( $\kappa=\kappa_{0} E^{\delta}$, with $\left.\delta \approx 0.5\right)$ was assumed to be independent of position in the Galaxy. Their findings are summarized in Figure 11. It is clear that the difference between the strong radial gradient of the source distribution and the observed weak CR gradient can only be explained if a very large CR halo is present. Stecker \& Jones (225) have

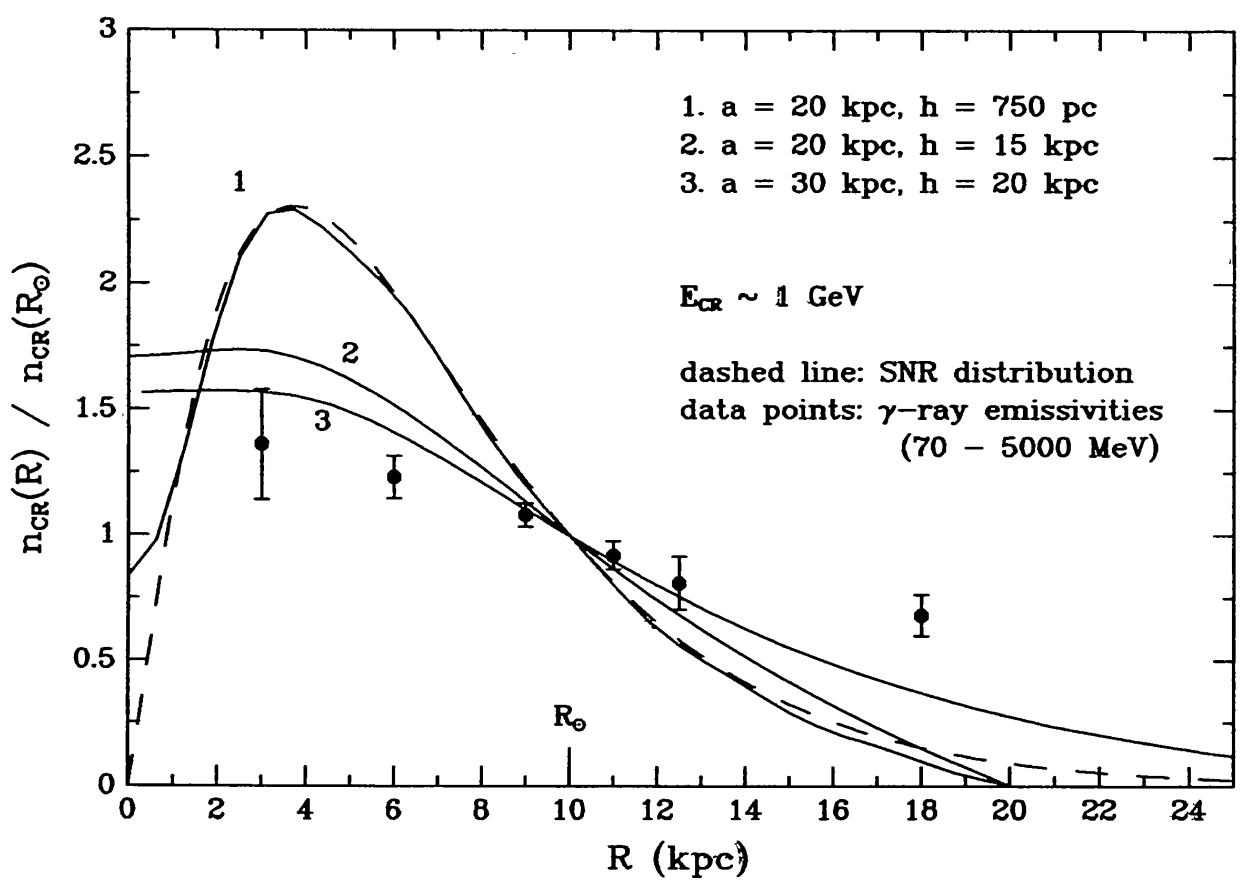

Figure 11 Examples of the Galacto-centric CR density distribution in a diffusion model with a supernova remnant-like CR source distribution and different halo dimensions ( $a$ : halo radius in the Galactic plane; $h$ : halo extent outside the plane). For details, see Bloemen $\&$ Dogiel (38). The data points indicate the $\gamma$-ray emissivity values derived by Strong et al. (232) (the energy-independent case, shown in Figure 5). All quantities are normalized at the radius of the solar circle. 
performed a similar analysis but concluded that thin halo models or sourcedominated diffusion models provide a good fit to the data. This conclusion was based, however, on the stronger radial gradient deduced from the early $S A S-2$ data base.

Wolfendale (253) has considered the possibility of an increase in the mean CR lifetime with increasing Galacto-centric distance as an explanation for the weak CR gradient, which may be related to the flaring of the gas disk in the outer Galaxy. In this scenario the shape of the CR confinement volume resembles that of the gas distribution, with the nearest escape boundary in the $z$-direction, so the distance to the CR escape boundary $D$ increases with increasing $R$; hence, in simple terms, the effective diffusion velocity $(v \propto \kappa / D)$ is smaller in the outer Galaxy and the mean CR lifetime $\left(\tau \propto D / v \propto D^{2} / \kappa\right)$ is larger.

As noted above, the energy dependence of the $\gamma$-ray model is in this case attributed to phenomena related to molecular clouds (or steep-spectrum $\gamma$-ray sources, distributed like the molecular gas). A soft $\gamma$-ray spectrum for molecular clouds is most simply explained by an enhanced electronto-proton ratio due to the production of secondary electrons [Brown \& Marscher $(45,163)$ ], which may be trapped [Cesarsky \& Völk (59)] and even further accelerated [Morfill $(175,176)]$. A useful review is given by Völk (248).

(b) ENERGY-DEPENDENT GRADIENT A possible energy dependence of the radial emissivity distribution can be attributed to a stronger gradient for the electrons than for the protons. In this case, the proton distribution is even harder to understand in the framework of a diffusion model. Also, there is no straightforward explanation for the energy dependence. Again, as in the scenario depicted above, differing CR lifetimes in the inner and outer Galaxy may play a role, but in this case owing to the fact that the relevant electrons have lower energies than the protons. This possibility was considered by Bhat et al. (15) and Bloemen (33). Measurements of the abundance ratios between $\mathrm{CR}$ secondaries and primaries indicate that locally the CR lifetime is approximately proportional to $E^{-0.5}$ above a few $\mathrm{GeV} /$ nucleon and flattens off at lower energies [see, e.g., the review by Wefel (252), and references therein]. Thus the stronger gradient for electrons than for protons (i.e. a stronger gradient for low-energy particles) may indicate that the CR lifetime falls with $E$ increasingly more slowly with increasing Galacto-centric distance; that is, in the inner Galaxy $\tau$ falls with $E$ more rapidly than $E^{-0.5}$, and in the outer Galaxy it falls more slowly. In view of the probably widely different structures of the ISM in the inner and outer Galaxy [e.g. Heiles (104)], such a different energy dependence of $\tau$ might be explicable. For instance, nonlinear wave damping 
(60), which produces a stronger energy dependence for the diffusion coefficient than the linear damping mechanism (140), may be important for $R \lesssim R_{\odot}$ but not in the outer Galaxy; this can possibly be ascribed to a radial falloff in the abundance of the highly ionized $\left(\sim 10^{4} \mathrm{~K}\right)$ medium (e.g. 139) in which the nonlinear mechanism may operate.

The $\gamma$-ray observations themselves, however, provide some counterevidence. If the energy dependence of the CR lifetime is indeed weaker in the outer Galaxy than in the inner Galaxy, this should show up in the $\gamma$-ray emission at high energies $(\gtrsim 300 \mathrm{MeV})$, which reflects directly the shape of the CR proton spectrum (Section 2.1). In other words, the $\gamma$-ray spectrum at high energies should be steeper in the inner-Galaxy direction than in the outer-Galaxy direction. As discussed at the end of Section 2.3, Bloemen (33) indeed found evidence for this effect (see Figure 7) but had to conclude from follow-up work (39) that the relative flatness of the average outer-Galaxy spectrum above $300 \mathrm{MeV}$ results from a gradual spectral flattening with increasing latitude (see Figure 8); near the Galactic plane, the high-energy $\gamma$-ray spectra of the inner and outer Galaxy are not significantly different.

Alternatively, the CR source spectrum may flatten with $R$, but here also a physical explanation is not evident.

It is clear from the above that option $(b)$ is hard to understand physically. Fortunately, as discussed in Section 2.3, the most recent tests prefer option (a), although only marginally.

The term "CR gradient" should be used with care because the observed $\gamma$-ray intensities trace predominantly CR particles in those regions along the line of sight that contribute significantly to the total gas column density. For instance, in the local Galactic environment more than $80 \%$ of the gas mass seems to be concentrated in clouds with a filling factor $<20 \%$, so practically no $\mathrm{CR}$ information can be obtained here from $\gamma$-ray observations for the major part of space. The $\mathrm{CR}$ density may be lower as well as higher in the intercloud region without being noticed by $\gamma$-ray observations. Hence, the radial gradient of the volume-averaged $\mathrm{CR}$ density may be stronger (or weaker) than that derived from $\gamma$-ray observations.

\subsection{Cosmic-Ray-Matter Coupling}

It has been argued on theoretical grounds that the CR density is correlated with the matter density, but the scale on which this correlation may occur is uncertain. Bignami \& Fichtel (22) have summarized some fundamental theoretical considerations and concluded that it may at least occur on the scale of spiral arms and large clouds. As discussed in Section 2.3, followup work showed that a model with CR-matter coupling on the scale of 
arms is indeed in agreement with $\gamma$-ray observations. Mainly the $S A S-2$ data have been analyzed this way, although Fichtel \& Kniffen (86) found reasonable agreement for the $C O S-B$ data as well. A radial gradient was not needed.

One can ask whether the above implies that the available $\gamma$-ray observations cannot distinguish between a "gradient model" and a "coupling model." Until recently, it was not very meaningful to compare the predictions from the two approaches because the coupling option was applied only to a model of the gas distribution, whereas studies of the gradient option made use of detailed $\mathrm{H} \mathrm{I}$ and $\mathrm{CO}$ surveys, taking into account all structures in the data. New insight follows from the preliminary results of a study by Melisse \& Bloemen (172), who are reconsidering CR-matter coupling models based on these surveys. They investigate whether the $C O S-B$ intensity distribution of the Milky Way can be described by a relation of the form

$$
I_{\gamma}=\varepsilon_{\gamma}\left(\mathbf{r}_{\odot}\right) \int_{l} n_{\mathrm{H}}(\mathbf{r})\left(\frac{n_{\mathrm{H}}(\mathbf{r})}{n_{\mathrm{H}}\left(\mathbf{r}_{\odot}\right)}\right)^{\alpha} d \mathbf{r},
$$

where $n_{\mathrm{H}}(\mathbf{r})$ is the gas distribution, derived from $\mathrm{CO}$ and $\mathrm{H} \mathrm{I}$ surveys, and the integration is along the line of sight. (Some modeling is required because of the distance-ambiguity problem.) The full velocity resolution of the surveys is used for the derivation of the gas densities, corresponding to length scales of typically 10-100 pc (i.e. scales of large clouds and cloud complexes). Free parameters are $\varepsilon_{\gamma}\left(\mathbf{r}_{\odot}\right), n_{\mathrm{H}}\left(\mathbf{r}_{\odot}\right), \alpha$, and the $N\left(\mathrm{H}_{2}\right)$-to- $W_{\mathrm{CO}}$ ratio $X$. One of the main aims of this work is to put constraints on the value of the $\alpha$ exponent in the $\rho_{\mathrm{CR}}-\rho_{\text {gas }}$ relationship $\left(\rho_{\mathrm{CR}} \propto \rho_{\mathrm{gas}}^{\alpha}\right)$, but this analysis has not been completed yet. As an example, a longitude profile of the modeled intensity distribution for $\alpha=0.5$ is included in Figure 3; in order to enable a direct comparison with the gradient model of Strong et al. (232), the same IC model has been added. This figure suggests that the coupling model fits the data not significantly worse than the gradient model-statistical tests will show whether this is indeed the case. On the other hand, the preliminary results indicate that fits with $\alpha \gtrsim 0.5$ become increasingly worse. It is too early for further discussion on this point.

\subsection{Large-Scale Spectral Variations}

We saw in Section 4.3 that the large-scale variation of the $\gamma$-ray spectrum along the Milky Way does not necessarily imply that the shape of the CR spectrum changes: The most plausible explanation for the relatively high $70-150 \mathrm{MeV}$ intensities in the inner-Galaxy direction is an enhanced electron density in molecular clouds. However, from the observed spectral 
variations of the $\gamma$-ray emission within the $300 \mathrm{MeV}-5 \mathrm{GeV}$ band (Section 2.3 ), it is very hard to avoid the conclusion that spectral variations for cosmic rays (protons) do exist. The hardening of the $\gamma$-ray spectrum with increasing latitude in the general direction of the outer Galaxy (39) suggests that the $\mathrm{CR}$ proton spectrum at $\mathrm{GeV}$ energies is flatter at medium latitudes, with a change in spectral index of $\sim 0.4-0.6$. Toward the inner Galaxy, this effect is not seen and the spectra are similar to that near the plane in the outer Galaxy (and consistent with direct measurements of the proton spectrum near Earth).

Bloemen \& Dogiel (38) have investigated an alternative possibilitynamely, whether the presence of an intense IC halo can explain the spectral behavior of the $\gamma$-ray data-triggered by the facts that a large CR halo may account for the weak CR gradient and that the IC $\gamma$-ray spectrum is relatively flat (Section 2.1). They found that the presence of an IC halo indeed leads to a hardening of the $\gamma$-ray spectrum with latitude; however, the observed effect is significantly stronger, and they could not explain the fact that it is only seen toward the outer Galaxy.

It is very interesting that a recent spectral analysis of radio-continuum surveys of the Galaxy (at 408 and $1420 \mathrm{MHz}$ ) by Reich \& Reich (197, 198) shows a similar spectral flattening with latitude in the outer-Galaxy direction. In this case, $\mathrm{GeV}$ electrons are traced; the change in the radio spectral index is about $0.2-0.25$, so the change in the electron spectral index is $\sim 0.4-0.5$ (if most of the radio emission has a nonthermal origin). Reich \& Reich found some evidence for a similar effect toward the inner Galaxy, but it was not possible to perform a reliable analysis here because only Northern Hemisphere data are available at $1420 \mathrm{MHz}$ and a large fraction of the available sky coverage is dominated by emission from Loop I. On the basis of radio-continuum data alone, a thermal origin of the flattening could not be excluded; however, this interpretation seems unlikely, since a similar effect is visible in the $\gamma$-ray data.

Although the interpretation of these findings is not straightforward, it is clear that a standard CR diffusion model, as described by Ginzburg \& Syrovatskii (95), encounters problems: A spectral steepening with distance from the Galactic plane would be expected for the electrons, due to synchrotron and Compton radiation losses, and no spectral changes for the protons. At low radio frequencies a weak spectral steepening with latitude was indeed found in previous work [see, e.g., the review by Lawson et al. (144)]. A dynamical halo model (Section 4.1) may provide a viable explanation. The competition of spatial diffusion, convection, adiabatic deceleration, and (electron) radiation losses in such a model may in principle lead to the observed effects, but the problem is very complex and requires robust modeling. Reich \& Reich (198) and Bloemen et al. (39) 
noted that the findings are to a large extent in agreement with the asymptotic spectral predictions of the Galactic wind model given by Lerche \& Schlickeiser $(152,153)$. Rogers et al. (204; see also 245, 246) have suggested that the spectral changes may have a local origin, related to our location on the inner edge of the Orion spiral arm and there being acceleration mechanisms at work within the arm such as to give a flatter CR spectrum inside the arm than in the interarm region. At the moment there certainly seems room for further speculation on the nature of this peculiar spectral behavior. Radio studies of other galaxies may shed new light on this topic in the near future.

\section{GAMMA-RAY SOURCES}

This section briefly reports on the status of searches for $\gamma$-ray point sources in the $C O S-B$ data base, mainly illustrating how considerable progress could (and still can) be made with the improved understanding of the diffuse emission from the Milky Way. A complete account of studies of individual sources is beyond the scope of this paper-a comprehensive review is given by Bignami \& Hermsen (24). I skip all discussion of the work on source models - it would be hopelessly incomplete in the space remaining-but remind the reader that $\gamma$-ray sources may be a manifestation of the presence of $\mathrm{CR}$ sources, such as the interaction of a supernova remnant with an adjacent gas cloud [see, e.g., the review by Morfill \& Tenorio-Tagle (177)]. The identification of such sources would enhance significantly our confidence in the interpretation of the large scale $\gamma$-ray distribution.

After some early claims based on balloon experiments, firm detection of $\gamma$-ray sources was first achieved with $S A S$-2: It confirmed the detection of $\gamma$ rays from the Crab pulsar $(134,243)$, discovered the intense $\gamma$-ray emission from the Vela pulsar $(241,244)$ and an hitherto puzzling source in the Galactic anticenter (243; now known as "Geminga"), and reported periodic emission from Cyg X-3 $(89,142)$. The latter result is under debate - it is not seen by $C O S-B$ (111). Already the first $C O S$ - $B$ observations showed evidence for several additional sources (114), which at that stage were simply defined as statistically significant peaks in the $\gamma$-ray intensity distribution, with an angular shape consistent with the $C O S-B$ point-spread function. An extensive account of the search for sources following this definition is given by Hermsen $(109,110)$; analysis of the data obtained during roughly the first half of the $\operatorname{COS}-B$ mission led to the so-called 2CG catalog (239), containing $25 \gamma$-ray sources, including the Crab and Vela pulsars and Geminga. Crab and Vela are unambiguously identified by their timing signatures; their temporal and spectral charac- 
teristics have been analyzed in great detail [see (62) and (97), respectively, and references therein]. There have also been searches for pulsed emission from other sources, but no firm detections have been obtained. All other identifications have to be based solely on positional coincidence. Given the limited angular resolution, it is difficult to differentiate between intrinsically compact objects and objects that might be up to a few degrees in size. Uniquely identifying counterparts at other wavelengths is therefore practically impossible, but two proposed identifications are very convincing: the quasar 3C 273 (e.g. 20, 112) and the $\rho$ Oph molecular cloud, which was in fact shown to be an extended $\gamma$-ray excess in subsequent work (113).

The unidentified sources of the 2CG catalog, except one (so 20 in total), are all located very close to the Galactic plane. This raised the question [one, in fact, already raised by Black \& Fazio (26) long before the sources were detected] as to whether a significant fraction of the $\gamma$-ray sources are possibly of diffuse origin, i.e. are associated with CR-irradiated clouds, or, more precisely, with peaks in the gas column-density distribution [see, e.g., Li Ti pei \& Wolfendale (159)]. With the availability of CO surveys it became feasible to take this diffuse emission into account in the search for sources. Therefore, the data obtained during the second half of the COS$B$ mission have never been used to update the $2 \mathrm{CG}$ catalog, applying the search method presented by Hermsen $(109,110)$. The new approaches are presented by Pollock et al. $(193,194)$ and Simpson \& Mayer-Hasselwander $(170,216)$; they consist of searching for $\gamma$-ray excesses, consistent with the $C O S-B$ point-spread function, superimposed on a model for the diffuse emission based on $\mathrm{H} \mathrm{I}$ and CO surveys, as described in Section 2.3. This implies a change in the original definition of a $\gamma$-ray source: Those original sources that find an explanation in terms of the same CR-matter interactions as the underlying diffuse emission are excluded. Remaining sources may of course still be of diffuse origin, possibly indicating an enhanced CR density somewhere along the line of sight.

Unfortunately, these new searches for $\gamma$-ray sources have not been completed yet. The results published so far suggest that about $30-50 \%$ of the 2CG sources can be attributed to structure in the gas distribution without requiring any $\mathrm{CR}$ density enhancement. On the other hand, there is some evidence for new sources. A 3CG catalog of $\gamma$-ray sources can soon be expected - possibly even more than one version, based on different methods for determining the statistical significance of the sources and the extraction of source parameters.

In order to leave the interested reader not entirely with empty hands at this stage, I have produced a "finding chart" of potential $\gamma$-ray sources for the energy range $100 \mathrm{MeV}-6 \mathrm{GeV}$ (Figure 12). Basically this map repre- 

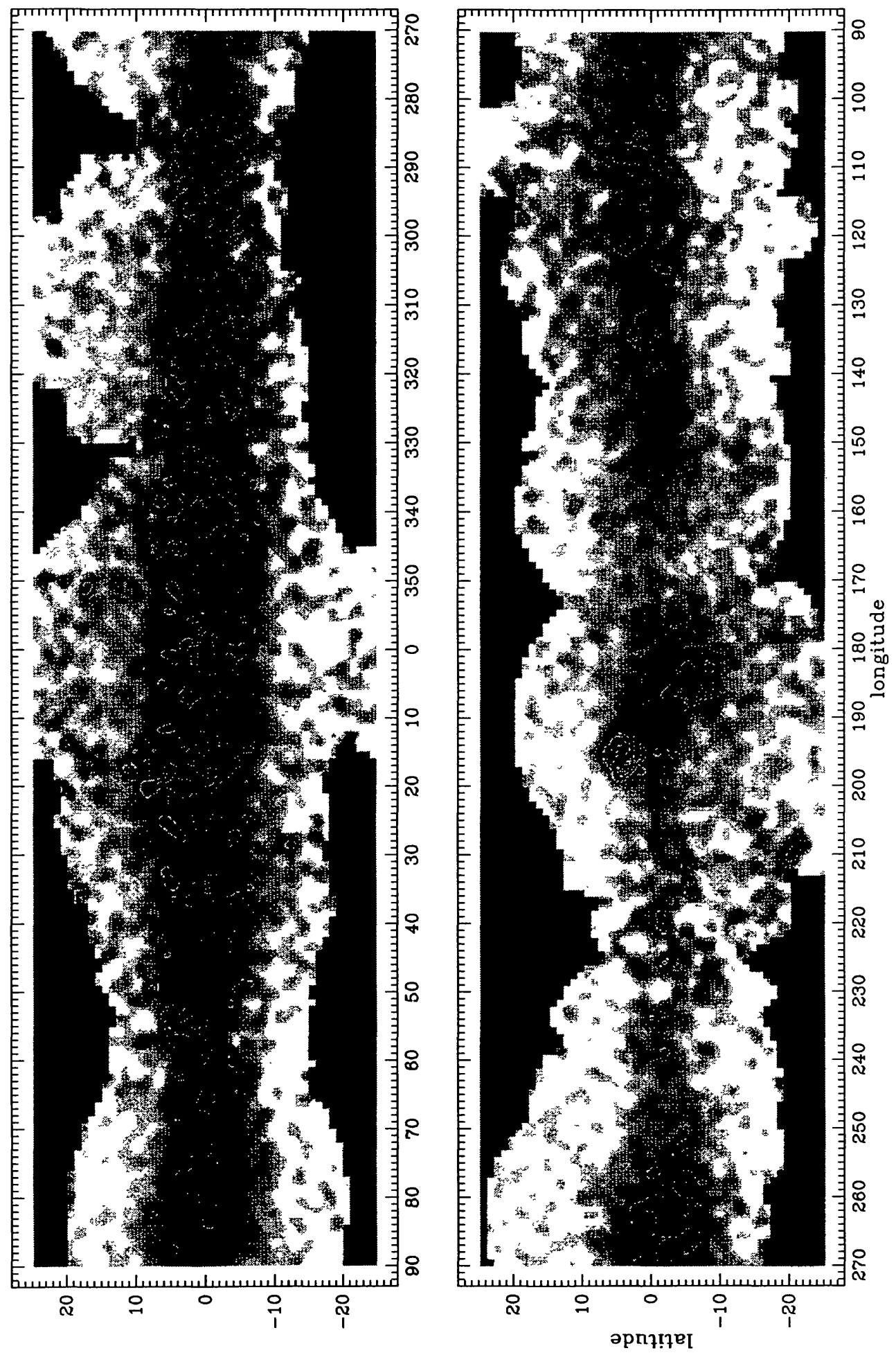

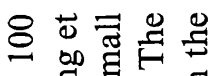

응

总部导兽

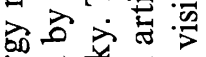

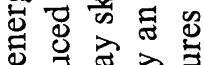

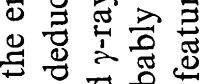

$\exists$ क

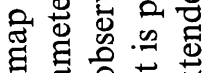

घ

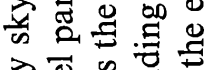

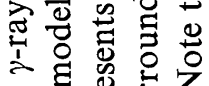

0 Z

मे

O 멸

要

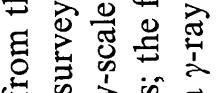

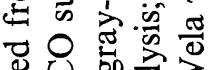

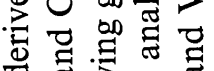

踏要

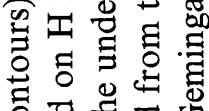

ठี

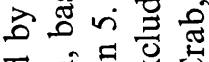

उี है.

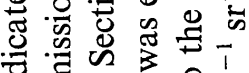

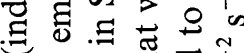

o

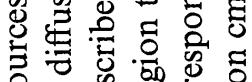

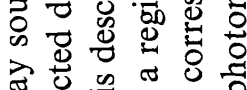

究曾.

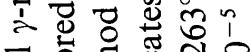

전 홍

힝.

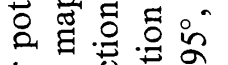

눙

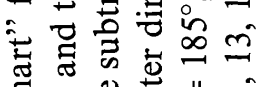

응

on

污寄兽焉

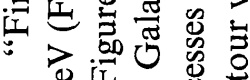

ح

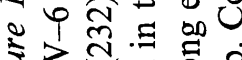

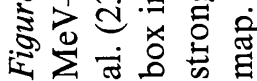


sents the $\gamma$-ray excesses that are left when the expected diffusion emission [based on the modeling of Strong et al. (232) (i.e. the map shown in Figure 4 , convolved with the $C O S-B$ point-spread function)] is subtracted from the observed $\gamma$-ray intensity map shown in Figure 1. However, it is not a pure subtraction. In order to correct for intermediate-scale deviations (10$20^{\circ}$ ) between model and observations, the model intensities have first been readjusted to the observed intensities on this scale: For a given position, the predictions in a strip of 21 pixels in longitude direction and 1 pixel in latitude (pixel size is $0.5^{\circ} \times 0.5^{\circ}$ ), centered on this position, were scaled upward or downward, such that for only $20 \%$ of the pixels are the observed intensities below the predicted intensities. Clearly, this is rather arbitrary, and no formal statistical significance can be assigned to the excesses visible in the finding chart. Interestingly, in addition to pointlike features, extended "sources" can be seen, with the most pronounced ones being the structures near $l \simeq 19^{\circ}$ (not coinciding with Loop I) and $l \simeq 334^{\circ}$, both of which extend over at least $10-15^{\circ}$. I leave speculations on their origin to the reader. These features may turn out to be the cream of the COS-B data base, just hinting at what the next generation of $\gamma$-ray telescopes may show us. By the time this review appears in print, the Soviet-French $\gamma$-ray telescope GAMMA-1 will hopefully be in orbit, and NASA's Gamma Ray Observatory should follow within half a year.

\section{ACKNOWLEDGMENTS}

I am grateful to V. A. Dogiel, V. L. Ginzburg, W. Hermsen, F. P. Israel, P. Maloney, A. W. Strong, and A. W. Wolfendale for critically reading the manuscript. In addition, I acknowledge the receipt of a Fellowship from the Royal Netherlands Academy of Arts and Sciences (KNAW) and partial support by the Laboratory for Space Research, Leiden.

\section{Literature Cited}

1. Allen, R. J., Baldwin, J. E., Sancisi, R. 1978. Astron. Astrophys. 62: 397

2. Arnaud, K., Li Ti pei, Riley, P. A., Wolfendale, A. W., Dame, T. M., et al. 1982. MNRAS 201: 745

3. Arnaud, K., Cassé, M. 1985. Astron. Astrophys. 144: 64

4. Audouze, J., Lequeux, J., Masnou, J. L., Puget, J. L. 1979. Astron. Astrophys. 80: 276

5. Axford, W. I. 1981. In Origin of Cosmic Rays, ed. G. Setti, G. Spada, A. W. Wolfendale, p. 339. Dordrecht: Reidel

6. Axford, W. I. 1987. Proc. Int. Cosmic Ray Conf., 20th, Moscow, 8: 120

7. Axford, W. I., Leer, E., Skandron, K.
G. 1977. Proc. Int. Cosmic Ray Conf., 15th, Plovdiv, 11: 132

8. Baldwin, J. E. 1976. In The Structure and Content of the Galaxy and Galactic Gamma Rays, ed. C. E. Fichtel, F. W. Stecker, p. 206. Greenbelt, Md: Goddard Space Flight Cent.

9. Beck, R., Biermann, P., Emerson, D. T., Wielebinski, R. 1979. Astron. Astrophys. 77: 25

10. Bell, A. R. 1978. MNRAS 182: 147

11. Berkhuijsen, E. M. 1971. Astron. Astrophys. 14: 359

12. Beuermann, K., Kanbach, G., Berkhuijsen, E. M. 1985. Astron. Astrophys. 153: 17 
13. Bhat, C. L., Issa, M. R., Houston, B. P., Mayer, C. J., Wolfendale, A. W. 1985. Nature 314: 511

14. Bhat, C. L., Issa, M. R., Mayer, C. J., Wolfendale, A. W. 1985. Nature 314: 515

15. Bhat, C. L., Mayer, C. J., Rogers, M., Wolfendale, A. W., Zan, M. 1986. J. Phys. G 12: 1087

16. Bhat, C. L., Mayer, C. J., Wolfendale, A. W. 1984. Astron. Astrophys. 140: 284

17. Bhat, C. L., Mayer, C. J., Wolfendale, A. W. 1986. Philos. Trans. R. Soc. London Ser. A 319: 249

18. Bignami, G. F. 1981. Philos. Trans. $R$. Soc. London Ser. A 301: 555

19. Bignami, G. F., Barbareschi, L., Bloemen, J. B. G. M., Buccheri, R., Caraveo, P. A., et al. 1981. Proc. Int. Cosmic Ray Conf., 17th, Paris, 1: 182

20. Bignami, G. F., Bennett, K., Buccheri, R., Caraveo, P. A., Hermsen, W., et al. 1981. Astron. Astrophys. 93: 71

21. Bignami, G. F., Boella, G., Burger, J. J., Keirle, P., Mayer-Hasselwander, $H$. A., et al. 1975. Space Sci. Instrum. 1: 245

22. Bignami, G. F., Fichtel, C. E. 1974. Ap. J. Lett. 189: L65

23. Bignami, G. F., Fichtel, C. E., Kniffen, D. A., Thompson, D. J. 1975. Ap. J. 199: 54

24. Bignami, G. F., Hermsen, W. 1983. Annu. Rev. Astron. Astrophys. 21: 67

25. Bignami, G. F., Piccinotti, G. 1977. Astron. Astrophys. 59: 233

26. Black, J. H., Fazio, G. G. 1973. Ap. J. Lett. 185: L7

27. Blandford, R. D., Ostriker, J. P. 1978. Ap. J. Lett. 221: L29

28. Blandford, R. D., Ostriker, J. P. 1980. Ap. J. 237: 793

29. Blandford, R. D., Cowie, L. L. 1982. Ap. J. 260: 625

30. Blitz, L., Bloemen, J. B. G. M. Hermsen, W., Bania, T. M. 1985. Astron. Astrophys. 143: 267

31. Bloemen, J. B. G. M. 1985. PhD thesis. Leiden Univ., Neth.

32. Bloemen, J. B. G. M. 1985. Astron. Astrophys. 145: 391

33. Bloemen, J. B. G. M. 1987. Ap. J. Lett. 317: L15

34. Bloemen, J. B. G. M. 1988. In Genesis and Propagation of Cosmic Rays, ed. M. M. Shapiro, J. P. Wefel, p. 163. Dordrecht: Reidel

35. Bloemen, J. B. G. M., Bennett, K., Bignami, G. F., Blitz, L., Caraveo, P. A., et al. 1984. Astron. Astrophys. 135: 12

36. Bloemen, J. B. G. M., Blitz, L., Hermsen, W. 1984. Ap. J. 279: 136
37. Bloemen, J. B. G. M., Caraveo, P. A., Hermsen, W., Lebrun, F., Maddalena, R. J., et al. 1984. Astron. Astrophys. 139: 37

38. Bloemen, J. B. G. M., Dogiel, V. A. 1989. In preparation

39. Bloemen, J. B. G. M., Reich, P., Reich, W., Schlickeiser, R. 1988. Astron. Astrophys. 204: 88

40. Bloemen, J. B. G. M., Strong, A. W., Blitz, L., Cohen, R. S., Dame, T. M., et al. 1986. Astron. Astrophys. 154: 25

41. Blumenthal, G. R., Gould, R. J. 1970. Rev. Mod. Phys. 42: 237

42. Brecher, K., Burbidge, G. R. 1972. Ap. J. 174: 253

43. Brindle, C., French, D. K., Osborne, J. L. 1978. MNRAS 184: 283

44. Bronfman, L., Cohen, R. S., Alvarez, H., May, J., Thaddeus, P. 1988. Ap. J. 324: 248

45. Brown, R. L., Marscher, A. P. 1977. Ap. J. 212: 659

46. Burbidge, G. R. 1974. Philos. Trans $R$. Soc. London Ser. A 277: 481

47. Burbidge, G. R. 1983. In Composition and Origin of Cosmic Rays, ed. M. M. Shapiro, p. 245. Dordrecht: Reidel

48. Burton, W. B. 1976. Annu. Rev. Astron. Astrophys. 14: 275

49. Burton, W. B. 1985. Astron. Astrophys. Suppl. 62: 365

50. Deleted in proof

51. Burton, W. B., te Lintel Hekkert, P. 1985. Astron. Astrophys. Suppl. 62: 645

52. Burton, W. B., te Lintel Hekkert, $\mathbf{P}$. 1986. Astron. Astrophys. Suppl. 65: 427

53. Caraveo, P. A., Bennett, K., Bignami, G. F., Hermsen, W., Kanbach, G. 1980. Astron. Astrophys. 91: L3

54. Cassé, M., Goret, P. 1978. Ap. J. 221: 703

55. Cesarsky, C. J. 1980. Annu. Rev. Astron. Astrophys. 18: 289

56. Cesarsky, C. J. 1987. Proc. Int. Cosmic Ray Conf., 20th, Moscow, 8: 87

57. Cesarsky, C. J., Cassé, M., Paul, J. A. 1977. Astron. Astrophys. 60: 139

58. Cesarsky, C. J., Paul, J. A., Shukla, P. G. 1978. Astrophys. Space Sci. 59: 73

59. Cesarsky, C. J., Völk, H. J. 1978. Astron. Astrophys. 70: 367

60. Chin, Y., Wentzel, D. G. 1972. Astrophys. Space Sci. 16: 465

61. Chupp, E. L. 1976. Gamma Ray Astronomy. Dordrecht: Reidel

62. Clear, J., Bennett, K., Buccheri, R., Grenier, I. A., Hermsen, W., et al. 1987. Astron. Astrophys. 174: 85

63. Clifton, T. R., Frail, D. A., Kulkarni, S. R., Weisberg, J. M. 1988. Ap. J. 333: 332 
64. Cowsik, R. 1980. Ap. J. 241: 1195

65. Cowsik, R. 1986. Astron. Astrophys. 155: 344

66. Cowsik, R., Wilson, L. W. 1975. Proc. Int. Cosmic Ray Conf., 14th, Munich, 2: 659

67. Dame, T. M., Elmegreen, B. G., Cohen, R. S., Thaddeus, P. 1986. Ap. J. 305: 892

68. Dame, T. M., Ungerechts, H., Cohen, R. S., de Geus, E., Grenier, I., et al. 1987. Ap. J. 322: 706

69. Dermer, C. D. 1986. Astron. Astrophys. 157: 223

70. Dickman, R. L., Snell, R. L., Schloerb, F. P. 1986. Ap. J. 309: 326

71. Dodds, D., Strong, A. W., Wolfendale, A. W. 1975. MNRAS 171: 569

72. Dogiel, V. A., Ginzburg, V. L. 1989. Space Sci. Rev. In press

73. Dogiel, V. A., Uryson, A. V. 1988. Astron. Astrophys. 197: 335

74. Draine, B. T., Salpeter, E. E. 1979. Ap. J. 231: 77

75. Draine, B. T., Salpeter, E. E. 1979. Ap. J. 231: 438

76. Eichler, D. 1979. Ap. J. 229: 419

77. Eichler, D. 1980. Ap. J. 237: 809

78. Ekers, R. D., Sancisi, R. 1977. Astron. Astrophys. 54: L973

79. Fazio, G. G. 1967. Annu. Rev. Astron. Astrophys. 5: 481

80. Fazio, G. G. 1973. In $X$ - and GammaRay Astronomy, ed. H. Bradt, R. Giacconi, p. 303. Dordrecht: Reidel

81. Fermi, E. 1954. Ap. J. 119: 1

82. Fichtel, C. E. 1977. Space Sci. Rev. 20: 191

83. Fichtel, C. E., Hartman, R. C., Kniffen, D. A., Thompson, D. J., Bignami, G. F., et al. 1975. Ap. J. 198: 163

84. Fichtel, C. E., Hartman, R. C., Kniffen, D. A., Thompson, D. J., Ögelman, H. B., et al. 1977. Ap. J. Lett. 217: L9

85. Fichtel, C. E., Hartman, R. C., Kniffen, D. A., Thompson, D. J., Ögelman, H. B., et al. 1978. NASA Tech. Memo. 79650

86. Fichtel, C. E., Kniffen, D. A. 1984. Astron. Astrophys. 134: 13

87. Fichtel, C. E., Kniffen, D. A., Thompson, D. J., Cheung, C. Y. 1976. Ap. J. 208: 211

88. Fichtel, C. E., Simpson, G. A., Thompson, D. J., 1978. Ap. J. 222: 833

89. Fichtel, C. E., Thompson, D. J., Lamb, R. C. 1987. Ap. J. 319: 362

90. Fichtel, C. E., Thrombka, J. I. 1981. Gamma-Ray Astrophysics, New Insight into the Universe. NASA SP-453

91. Fuchs, B., Schlickeiser, R., Thielheim, K. O. 1976. Ap. J. 206: 589
92. Ginzburg, V. L. 1953. Usp. Fiz. Nauk 51: 343

93. Ginzburg, V. L., ed. 1984. Cosmic-Ray Astrophysics. Moscow: Nauka (Engl. translation: 1989, North-Holland)

94. Ginzburg, V. L. 1987. Proc. Cosmic Ray Conf., 20th, Moscow, 7: 7

95. Ginzburg, V. L., Syrovatskii, S. I. 1964. The Origin of Cosmic Rays. Oxford: Pergamon

96. Gould, R. J. 1969. Phys. Rev. 185: 72

97. Grenier, I. A., Hermsen, W., Clear, J. 1988. Astron. Astrophys. 204: 117

98. Gualandris, F. L., Strong, A. W. 1984. Astron. Astrophys. 140: 357

99. Harding, A. K., Stecker, F. W. 1985. Ap. J. 291: 471

100. Hartman, R. C., Kniffen, D. A., Thompson, D. J., Fichtel, C. E., Ögelman, H. B., et al. 1979. Ap. J. 230: 597

101. Hayakawa, S. 1952. Prog. Theor. Phys. 8: 517

102. Hayakawa, S. 1969. Cosmic Ray Physics. New York: Wiley-Interscience

103. Heiles, C. 1987. In Interstellar Processes, ed. D. J. Hollenbach, H. A. Thronson, p. 171. Dordrecht: Reidel

104. Heiles, C. 1988. Ap. J. 324: 321

105. Heiles, C., Cleary, M. N. 1979. Aust. J. Phys. Suppl. 47: 1

106. Heiles, C., Habing, H. J. 1974. Astron. Astrophys. Suppl. 14: 1

107. Heiligman, G. 1982. $\mathrm{PhD}$ thesis. Princeton Univ., Princeton, N.J.

108. Henderson, A. P., Jackson, P. D., Kerr, F. J. 1982. Ap. J. 263: 182

109. Hermsen, W. 1980. PhD thesis. Leiden Univ., Neth.

110. Hermsen, W. 1983. Space Sci. Rev. 36: 61

111. Hermsen, W., Bennett, K., Bloemen, J. B. G. M., Buccheri, R., Jansen, F. A., et al. 1987. Astron. Astrophys. 175: 141

112. Hermsen, W., Bennett, K., Bignami, G. F., Bloemen, J. B. G. M., Buccheri, R., et al. 1981. Proc. Int. Cosmic Ray Conf., 17th, Paris, 1: 230

113. Hermsen, W., Bloemen, J. B. G. M. 1983. In Surveys of the Southern Galaxy, ed. W. B. Burton, F. P. Israel, p. 65. Dordrecht: Reidel

114. Hermsen, W., Swanenburg, B. N., Bignami, G. F., Boella, G., Buccheri, R., et al. 1977. Nature 269: 494

115. Higdon, J. C. 1979. Ap. J. 232: 113

116. Houston, B. P., Wolfendale, A. W. 1983. Astron. Astrophys. 126: 22

117. Houston, B. P., Wolfendale, A. W. 1985. J. Phys. G 11: 407

118. Hummel, E., Sancisi, R., Ekers, R. D. 1984. Astron. Astrophys. 133: 1

119. Hummel, E., Smith, P., van der Hulst, 
J. M. 1984. Astron. Astrophys. 137: 138

120. Hutchinson, G. W. 1952. Philos. Mag. 43: 847

121. Ipavich, F. 1975. Ap. J. 196: 107

122. Issa, M. R., Riley, P. A., Strong, A. W., Wolfendale, A. W. 1981. J. Phys. $G$ 7: 973

123. Issa, M. R., Strong, A. W., Wolfendale, A. W. 1981. J. Phys. G 7: 565

124. Issa, M. R., Wolfendale, A. W. 1981. J. Phys. G 7: L187

125. Issa, M. R., Wolfendale, A. W. 1981. Nature 292: 430

126. Jokipii, J. R. 1976. Ap. J. 208: 900

127. Jones, F. C. 1979. Ap. J. 229: 747

128. Kanbach, G., Bennett, K., Bignami, G. F., Buccheri, R., Caraveo, P. A., et al. 1980. Astron. Astrophys. 90: 163

129. Klein, R., Urbanik, M., Beck, R., Wielebinski, R. 1983. Astron. Astrophys. 127: 177

130. Klein, R., Wielebinski, R., Beck, R. 1984. Astron. Astrophys. 133: 19

131. Kniffen, D. A., Fichtel, C. E. 1981. Ap. J. 250: 389

132. Kniffen, D. A., Fichtel, C. E., Thompson, D. J. 1977. Ap. J. 215: 765

133. Kniffen, D. A., Hartman, R. C., Thompson, D. J., Bignami, G. F., Fichtel, C. E., et al. 1973. Ap. J. Lett. 186: L105

134. Kniffen, D. A., Hartman, R. C., Thompson, D. J., Bignami, G. F., Fichtel, C. E., et al. 1974. Nature 251: 397

135. Kodaira, K. 1974. Publ. Astron. Soc. Jpn. 26: 255

136. Kóta, J., Owens, A. J. 1980. Ap. J. 237: 814

137. Kraushaar, W. L., Clark, G. W., Garmire, G. P., Borken, R., Higbie, P., et al. 1972. Ap. J. 177: 341

138. Krymsky, G. F. 1977. Dokl. Akad. Nauk SSSR 234: 1306

139. Kulkarni, S. R., Heiles, C. 1987. In Interstellar Processes, ed. D. J. Hollenbach, H. A. Thronson, p. 87. Dordrecht: Reidel

140. Kulsrud, R. M., Pearce, W. P. 1969. Ap. J. 156: 445

141. Kutner, M. L., Leung, C. M. 1985. Ap. J. 291: 188

142. Lamb, R. C., Fichtel, C. E., Hartman, R. C., Kniffen, D. A., Thompson, D. J. 1977. Ap. J. Lett. 212: L63

143. Larson, R. B. 1981. MNRAS 194: 809

144. Lawson, K. D., Mayer, C. J., Osborne, J. L., Parkinson, M. L. 1987. MNRAS 225: 307

145. Lebrun, F. 1984. In Nearby Molecular Clouds. Lect. Notes Phys., ed. G. Serra, 237: 3. Berlin: Springer-Verlag

146. Lebrun, F. 1986. Ap. J. 306: 16
147. Lebrun, F., Bennett, K., Bignami, G. F., Bloemen, J. B. G. M., Buccheri, R., et al. 1983. Ap. J. 281: 634

148. Lebrun, F., Bignami, G. F., Buccheri, R., Caraveo, P. A., Hermsen, W., et al. 1982. Astron. Astrophys. 107: 390

149. Lebrun, F., Paul, J. A. 1983. Ap. J. 266: 276

150. Lebrun, F., Paul, J. A. 1985. Proc. Int. Cosmic Ray Conf., 19th, La Jolla, 1: 309

151. Lequeux, J. 1981. Comments Astrophys. 9: 117

152. Lerche, I., Schlickeiser, R. 1982. MNRAS 201: 1041

153. Lerche, I., Schlickeiser, R. 1982. Astron. Astrophys. 107: 148

154. Lester, D. F., Dinerstein, H. L., Werner, M. W., Watson, D. M., Genzel, R., Storey, J. W. V. 1987. Ap. J. 320: 573

155. Linke, R. A., Stark, A. A., Frerking, M. A. 1981. Ap. J. 243: 147

156. Liszt, H. S. 1984. Comments Astrophys. 10: 137

157. Li Ti pei, Riley, R. A., Wolfendale, A. W. 1982. J. Phys. G 8: 1141

158. Li Ti pei, Wolfendale, A. W. 1981. Astron. Astrophys. 100: L26

159. Li Ti pei, Wolfendale, A. W. 1981. Astron. Astrophys. 103: 19

160. Lyne, A. G., Manchester, R. N., Taylor, J. H. 1985. MNRAS 213: 613

161. MacLaren, I., Richardson, K. M., Wolfendale, A. W. 1988. Ap. J. 333: 821

162. Maloney, P., Black, J. 1988. Ap. J. 325: 389

163. Marscher, A. P., Brown, R. L. 1978. Ap. J. 221: 583

164. Mathis, J. S. 1986. Ap. J. 301: 423

165. Mathis, J. S., Mezger, P. G., Panagia, N. 1983. Astron. Astrophys. 128: 212

166. Mayer, C. J., Richardson, K. M., Rogers, M. J., Szabelski, J., Wolfendale, A. W. 1987. Astron. Astrophys. 180: 73

167. Mayer-Hasselwander, H. A. 1983. In Kinematics, Dynamics, and Structure of the Milky Way, ed. W. L. H. Shuter, p. 223. Dordrecht: Reidel

168. Mayer-Hasselwander, H. A. 1985. Explanatory Supplement to the COS-B Database (available from K. Bennett, SSD, ESTEC, Noordwijk, Neth.)

169. Mayer-Hasselwander, H. A., Bennett, K., Bignami, G. F., Buccheri, R., Caraveo, P. A., et al. 1982. Astron. Astrophys. 105: 164

170. Mayer-Hasselwander, H. A., Simpson, G. 1989. Adv. Space Res. In press

171. McKee, C. F., Hollenbach, D. J., Seab, 
C. G., Tielens, A. G. G. M. 1987. Ap. J. 318: 674

172. Melisse, J., Bloemen, J. B. G. M. 1989. In preparation

173. Meyer, J.-P. 1985. Ap. J. Suppl. 57: 173

174. Meyers, P. C. 1983. Ap. J. 270: 105

175. Morfill, G. E. 1982. MNRAS 198: 583

176. Morfill, G. E. 1982. Ap. J. 262: 749

177. Morfill, G. E., Tenorio-Tagle, G. 1983. Space Sci. Rev. 36: 93

178. Deleted in proof

179. Müller, D., Tang, J. 1981. Proc. Int. Cosmic Ray Conf., 17th, Paris, 9: 142

180. Nishimura, J., Fujii, M., Taira, T., Aiza, E., Hiraiwa, H., et al. 1980. Ap. J. 238: 394

181. Oort, J. H. 1977. Annu. Rev. Astron. Astrophys. 15: 295

182. Owens, A. J., Jokipii, J. R. 1977. Ap. J. 215: 677

183. Pagel, B. E. J., Edmunds, M. G. 1981. Annu. Rev. Astron. Astrophys. 19: 77

184. Parker, E. N. 1966. Ap. J. 145: 811

185. Parker, E. N. 1969. Space Sci. Rev. 9: 651

186. Parker, E. N. 1976. In The Structure and Content of the Galaxy and Galactic Gamma Rays, ed. C. E. Fichtel, F. W. Stecker, p. 320. Greenbelt, Md: Goddard Space Flight Cent.

187. Paul, J. A., Cassé, M., Cesarsky, C. J. 1976. Ap. J. 207: 62

188. Peters, B., Westergaard, N. J. 1977. Astrophys. Space Sci. 48: 21

189. Phillipps, S., Kearsey, S., Osborne, J. L., Haslam, C. G. T., Stoffel, H. 1981. Astron. Astrophys. 98: 286

190. Phillipps, S., Kearsey, S., Osborne, J. L., Haslam, C. G. T., Stoffel, H. 1981. Astron. Astrophys. 103: 405

191. Piccinotti, G., Bignami, G. F. 1976. Astron. Astrophys. 52: 69

192. Pikelner, S. B. 1953. Dokl. Acad. Sci. USSR 88: 229

193. Pollock, A. M. T., Bennett, K., Bignami, G. F., Bloemen, J. B. G. M., Buccheri, R., et al. 1985. Astron. Astrophys. 146: 352

194. Pollock, A. M. T., Bennett, K., Bignami, G. F., Bloemen, J. B. G. M., Buccheri, R., et al 1985. Proc. Int. Cosmic Ray Conf., 19th, La Jolla, 1: 338

195. Ptuskin, V. S. 1979. Astrophys. Space Sci. 61: 259

196. Ramana Murthy, P. V., Wolfendale, A. W. 1986. Gamma-Ray Astronomy. Cambridge: Univ. Press

197. Reich, P., Reich, W. 1988. Astron. Astrophys. Suppl. 74: 7

198. Reich, P., Reich, W. 1988. Astron. Astrophys. 196: 211

199. Richardson, K. M., Wolfendale, A. W. 1988. Astron. Astrophys. 201: 100
200. Richardson, K. M., Wolfendale, A. W. 1988. Astron. Astrophys. 203: 289

201. Riley, P. A., Wolfendale, A. W. 1984. J. Phys. G 10: 1269

202. Riley, P. A., Wolfendale, A. W., Xu, C.-x., Manchester, R. N., Robinson, B. J., Whiteoak, J. B. 1984. MNRAS 206: 423

203. Rockstroh, J. M., Webber, W. R. 1978. Ap. J. 224: 677

204. Rogers, M. J., Sadzinska, M., Szabelski, J., van der Walt, D. J., Wolfendale, A. W. 1988. J. Phys. G 14: 1147

205. Rogers, M. J., Wolfendale, A. W. 1987. Proc. Int. Cosmic Ray Conf., 20th, Moscow, 1: 81

206. Ryan, M. J., Balasubrahmanyan, V. K., Ormes, J. F. 1972. Phys. Rev. Lett. 28: 985

207. Sanders, D. B., Scoville, N. Z., Solomon, P. M. 1985. Ap. J. 289: 373

208. Sanders, D. B., Solomon, P. M., Scoville, N. Z. 1984. Ap. J. 276: 182

209. Scarsi, L., Bennett, K., Bignami, G. F., Boella, G., Buccheri, R., et al. 1977. Proc. 12th ESLAB Symp. ESA SP 124, p. 3

210. Scott, J. S. 1975. Nature 258: 58

211. Seldner, M., Siebers, B., Groth, E. J., Peebles, P. J. E. 1977. Astron. J. 82: 249

212. Shane, C. D., Wirtanen, C. A. 1967. Publ. Lick Obs. 22: 1

213. Shklovskii, I. S. 1952. Astron. Zh. 29: 418

214. Shukla, P. G., Paul, J. A. 1976. Ap. J. 208: 893

215. Silk, J., Bloemen, J. B. G. M. 1987. Ap. J. Lett. 313: L47

216. Simpson, G., Mayer-Hasselwander, H. A. 1987. Proc. Int. Cosmic Ray Conf., 20th, Moscow, 1: 89

217. Simpson, J. A. 1983. Annu. Rev. Nucl. Part. Sci. 33: 323

218. Skilling, J., Strong, A. W. 1976. Astron. Astrophys. 53: 253

219. Sofue, Y., Fujimoto, M., Wielebinski, R. 1986. Annu. Rev. Astron. Astrophys. 24: 459

220. Solomon, P. M., Rivolo, A. R., Barrett, J., Yahil, A. 1987. Ap. J. 319: 730

221. Stacy, J. G., Dame, T. M., Thaddeus, P. 1987. Proc. Int. Cosmic Ray Conf., 20th, Moscow, 1: 117

222. Stecker, F. W. 1971. Cosmic Gamma Rays. Baltimore: Mono Book Corp.

223. Stecker, F. W. 1973. Ap. J. 185: 499

224. Stecker, F. W. 1977. Ap. J. 212: 60

225. Stecker, F. W., Jones, F. C. 1977. Ap. J. 217: 843

226. Stecker, F. W., Solomon, P. M., Scoville, N. Z., Ryter, C. E. 1975. Ap. J. 201: 90 
227. Stephens, S. A., Badhwar, G. D. 1981. Astrophys. Space Sci. 76: 213

228. Strong, A. W. 1985. Astron. Astrophys. 145: 81

229. Strong, A. W. 1985. Proc. Int. Cosmic Ray Conf., 19th, La Jolla, 1: 333

230. Strong, A. W., Bennett, K., Bignami, G. F., Bloemen, J. B. G. M., Buccheri, R., et al. 1983. Proc. Int. Cosmic Ray Conf., 18th, Bangalore, 9: 90

231. Strong, A. W., Bignami, G. F., Bloemen, J. B. G. M., Buccheri, R., Caraveo, P. A., et al. 1982. Astron. Astrophys. 115: 404

232. Strong, A. W., Bloemen, J. B. G. M., Dame, T. M., Grenier, I., Hermsen, W., et al. 1988. Astron. Astrophys. 207: 1

233. Strong, A. W., Bloemen, J. B. G. M., Hermsen, W., Lebrun, F., MayerHasselwander, H. A. 1987. Astron. Astrophys. Suppl. 67: 283

234. Strong, A. W., Bloemen, J. B. G. M., Hermsen, W., Mayer-Hasselwander, H. A. 1985. Proc. Int. Cosmic Ray Conf., 19th, La Jolla, 1: 317

235. Deleted in proof

236. Strong, A. W., Wolfendale, A. W. 1978. J. Phys. G 4: 1

237. Strong, A. W., Wolfendale, A. W. 1981. Philos. Trans. R. Soc. London Ser. A 301: 541

238. Strong, A. W., Wolfendale, A. W., Bennett, K., Wills, R. D. 1978. MNRAS 182: 751

239. Swanenburg, B. N., Bennett, K., Bignami, G. F., Buccheri, R., Caraveo, P. A., et al. 1981. Ap. J. Lett. 243: L69

240. Szabelski, J., van der Walt, D. J., Wdowczyk, J., Wolfendale, A. W. 1989. Adv. Space Res. In press

241. Thompson, D. J., Bignami, G. F., Fich- tel, C. E., Kniffen, D. A. 1974. Ap. J.

Lett. 190: L51

242. Thompson, D. J., Fichtel, C. E. 1982. Astron. Astrophys. 109: 352

243. Thompson, D. J., Fichtel, C. E., Hartman, R. C., Kniffen, D. A., Lamb, R. C. 1977. Ap. J. 213: 252

244. Thompson, D. J., Fichtel, C. E., Kniffen, D. A., Ögelman, H. B. 1975. Ap. J. Lett. 200: L79

245. van der Walt, D. J., Wolfendale, A. W. 1988. J. Phys. G 14: L159

246. van der Walt, D. J., Wolfendale, A. W. 1988. Space Sci. Rev. 47: 1

247. Verter, F. 1987. Ap. J. Suppl. 65: 555

248. Völk, H. J. 1983. Space Sci. Rev. 36: 3

249. Völk, H. J. 1984. In High Energy Astrophysics, ed. J. Audouze, J. Tran Thanh Van, p. 281. Gif-sur-Yvette, Fr: Editions Frontières

250. Webber, W. R. 1983. In Composition and Origin of Cosmic Rays, ed. M. M. Shapiro, p. 83. Dordrecht: Reidel

251. Webber, W. R., Simpson, G. A., Cane, H. V. 1980. Ap. J. 236: 448

252. Wefel, J. P. 1988. In Genesis and Propagation of Cosmic Rays, ed. M. M. Shapiro, J. P. Wefel, p. 1. Dordrecht: Reidel

253. Wolfendale, A. W. 1986. In Cosmic Radiation in Contemporary Astrophysics, ed. M. M. Shapiro, p. 135. Dordrecht: Reidel

254. Wolfendale, A. W. 1988. In Molecular Clouds in the Milky Way and External Galaxies, ed. R. Dickman, R. Snell, J. Young, p. 76. Heidelberg: SpringerVerlag

255. Wolfendale, A. W. 1989. In Cosmic Gamma Rays and Cosmic Neutrinos, ed. M. M. Shapiro, J. P. Wefel. Dordrecht: Reidel. In press 